Prepared in collaboration with Wildlife Conservation Society Canada, USDA Forest Service, U.S. Army Corps of Engineers, Illinois Natural History Survey, New York State Department of Environmental Conservation, Colorado Natural Heritage Program, Montana Natural Heritage Program, National Park Service, and Bat Call Identification, Inc.

\title{
A Guide to Processing Bat Acoustic Data for the North American Bat Monitoring Program (NABat)
}
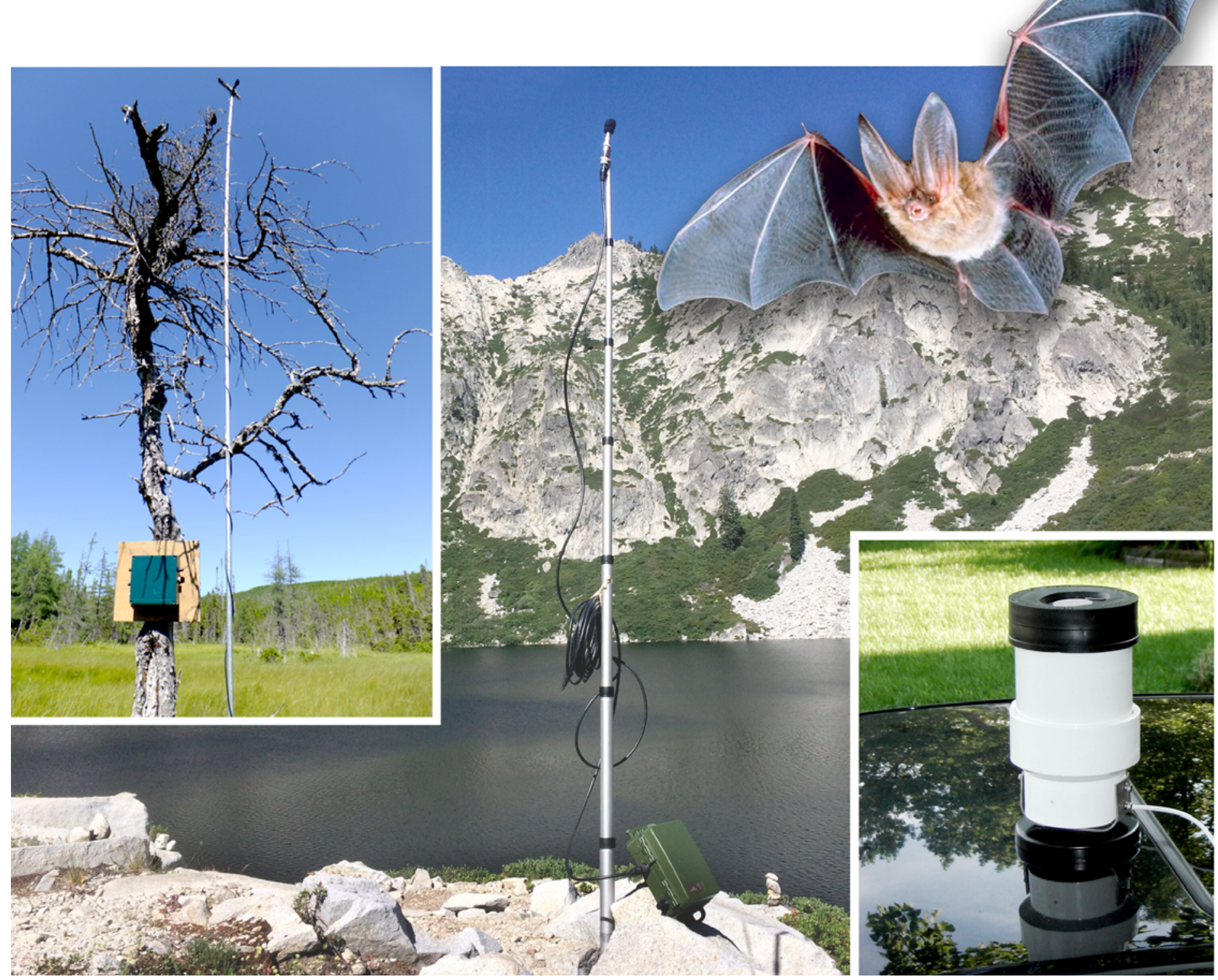

Open-File Report 2018-1068

U.S. Department of the Interior

U.S. Geological Survey 
Cover. Upper left: Fen (wetland) setup in western Newfoundland. Photograph by Jessica Humber, Department of Fisheries \& Land Resources, Government of Newfoundland and Labrador, Canada, 2016. Used with permission. Center: A detector in the Trinity Alps Wilderness Area, Shasta-Trinity National Forest, Calif. Photograph by T.J. Weller, U.S. Dept. of Agriculture (USDA) Forest Service, July 18, 2017. Lower right: A roof detector mount. Photograph by Carl Herzog, New York State Department of Environmental Conservation, 2015. Upper right: Townsend's big-eared bat (Corynorhinus townsendii), Wikipedia Commons, 2002, accessed May 2018, at https://commons.wikimedia.org/wiki/ File:Big-eared-townsend-fledermaus.jpg. 


\section{A Guide to Processing Bat Acoustic Data for the North American Bat Monitoring Program (NABat)}

By Brian Reichert, Cori Lausen, Susan Loeb, Ted Weller, Ryan Allen, Eric Britzke, Tara Hohoff, Jeremy Siemers, Braden Burkholder, Carl Herzog, and Michelle Verant

Prepared in collaboration with Wild life Conservation Society Canada, USDA Forest Service, U.S. Army Corps of Engineers, Illinois Natural History Survey, New York State Department of Environmental Conservation, Colorado Natural Heritage Program, Montana Natural Heritage Program, National Park Service, and Bat Call Identification, Inc.

Open-File Report 2018-1068 


\title{
U.S. Department of the Interior \\ RYAN K. ZINKE, Secretary
}

\author{
U.S. Geological Survey \\ James F. Reilly II, Director
}

U.S. Geological Survey, Reston, Virginia: 2018

For more information on the USGS - the Federal source for science about the Earth, its natural and living resources, natural hazards, and the environment-visit https://www.usgs.gov or call 1-888-ASK-USGS.

For an overview of USGS information products, including maps, imagery, and publications,

visit https://store.usgs.gov.

Any use of trade, firm, or product names is for descriptive purposes only and does not imply endorsement by the U.S. Government.

Although this information product, for the most part, is in the public domain, it also may contain copyrighted materials as noted in the text. Permission to reproduce copyrighted items must be secured from the copyright owner.

Suggested citation:

Reichert, B., and Lausen, C., Loeb, S., Weller, T., Allen, R., Britzke, E., Hohoff, T., Siemers, J., Burkholder, B., Herzog, C., and Verant, M., 2018, A Guide to processing bat acoustic data for the North American Bat Monitoring Program (NABat): U.S. Geological Survey Open-File Report 2018-1068, 33 p., https://doi.org/10.3133/ofr20181068.

ISSN 2331-1258 (online) 


\section{Acknowledgments}

We thank the bat community for their unwavering support of the North American Bat Monitoring Program. We especially thank David Riggs (Myotisoft, LLC), lan Agranat (Wildlife Acoustics, Inc.), Joe Szewczak (Humboldt State University, SonoBat), Chris Corben (Titley Scientific), Jenny Barnett (U.S. Fish and Wildlife Service), and François Fabianek (WavX, Inc. \& Groupe Chiroptères du Québec) for thoughtful discussion, suggestions, and comments on processing bat acoustic data. Janet Tyburec (Bat Survey Solutions, LLC) and Han Li (University of North Carolina at Greensboro) reviewed the manuscript and provided suggestions which greatly helped to improve this document. We also thank Janet Tyburec for contributing information on standardized codes for metadata.

\section{Contents}

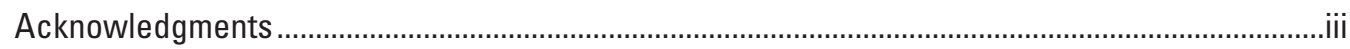

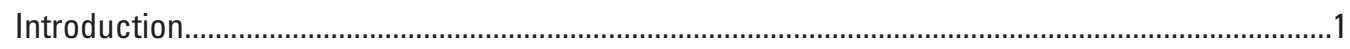

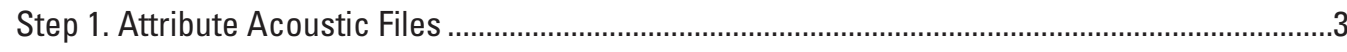

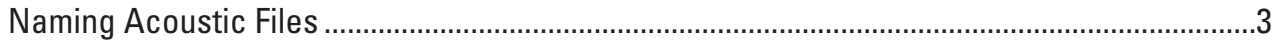

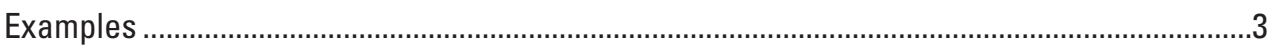

Assigning Metadata to Acoustic Files ...............................................................................

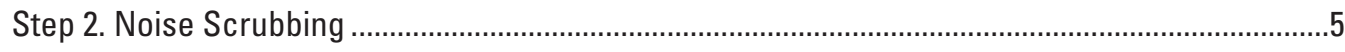

Scrubbing Stationary versus Mobile Datasets .....................................................................

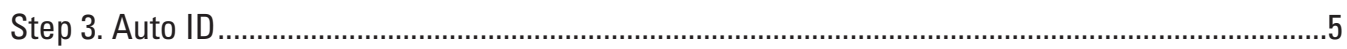

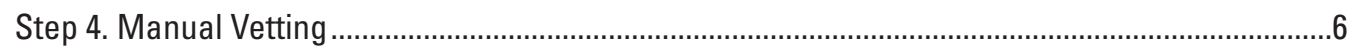

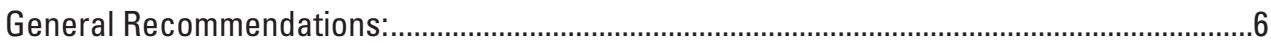

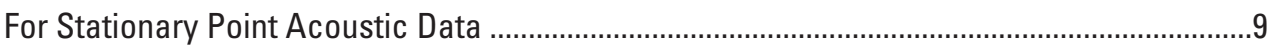

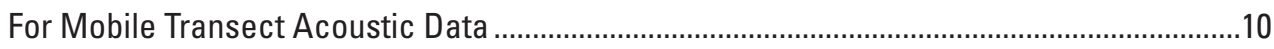

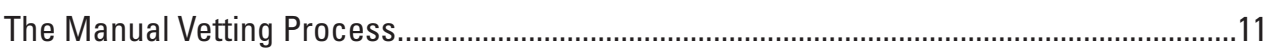

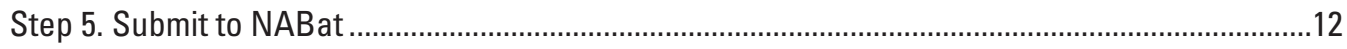

Chapter 1. Processing Acoustic Data Using Kaleidoscope Pro Version 4.3.2 …..............................13

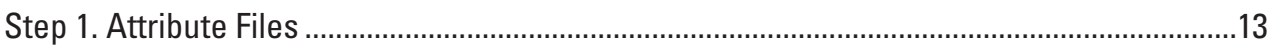

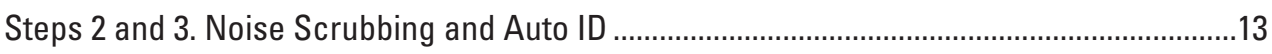

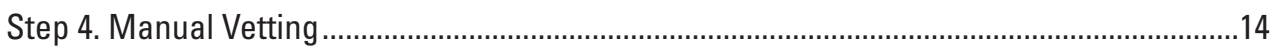

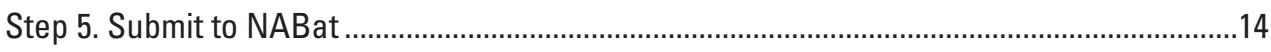

Chapter 2. Processing Full Spectrum Acoustic Data Using SonoBat 4.2.1 ...................................15

Step 1. Attribute Files Using SonoBat Attributer ..............................................................15

Step 2. Noise Scrubbing using SonoBat Scrubber and Attributer .........................................15

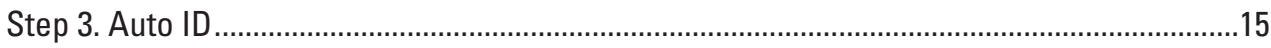

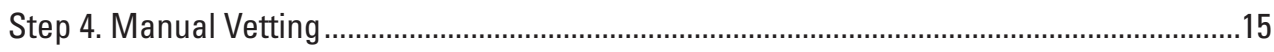

Stationary Point and Mobile Transect Data ……………………………………........16

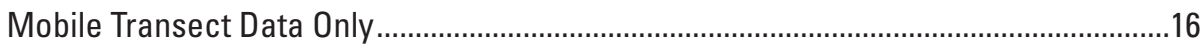

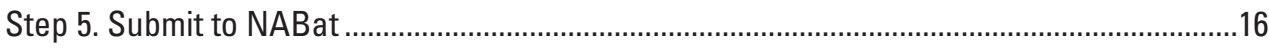

Chapter 3. Processing Acoustic Data Using Bat Call Identification ver. 2.7d (BCID) .......................17

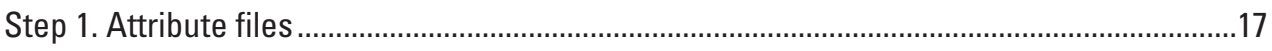

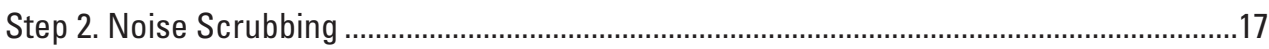




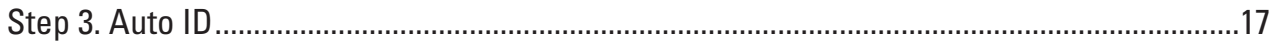

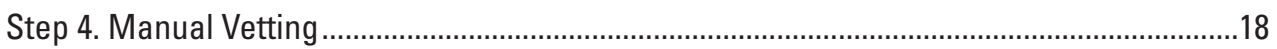

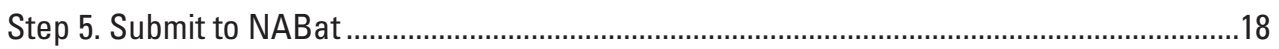

Chapter 4. Processing Zero-Cross Acoustic Data Using EchoClass v 3.1 ......................................19

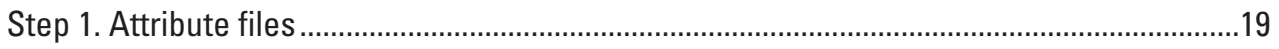

Tips for organizing your data........................................................................................

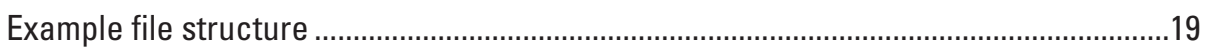

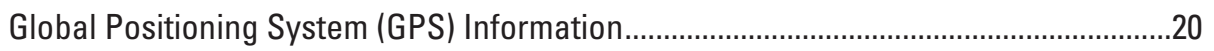

Mobile Transect Acoustic Monitoring..........................................................................20

Stationary Point Acoustic Monitoring ...................................................................20

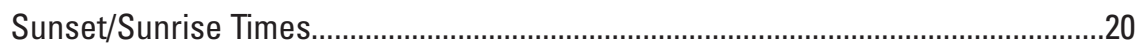

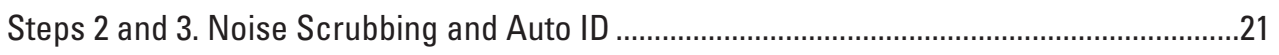

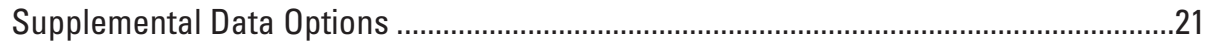

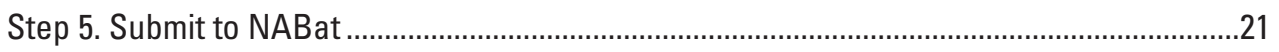

Chapter 5. Processing Zero-Cross Acoustic Data Using AnaLookW 4.2n

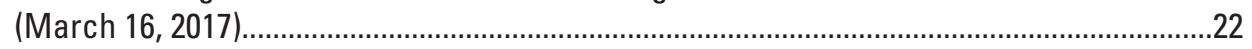

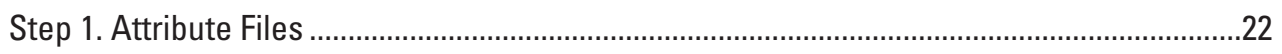

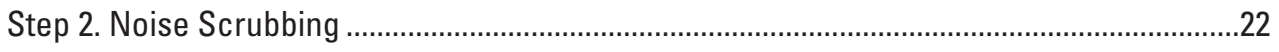

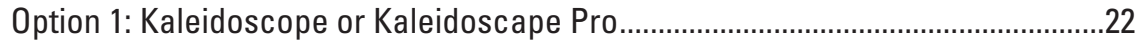

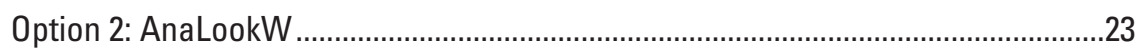

Step 3. Auto ID .................................................................................................................23

Option 1: Species Labels are Embedded in Files as MetaData During the Auto ID

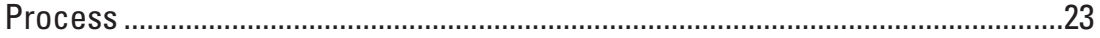

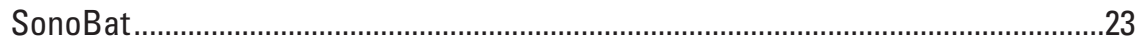

Kaleidoscope Pro

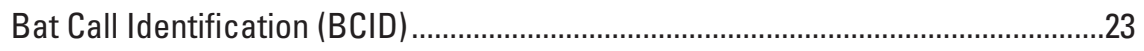

Option 2: Species Labels Are Embedded in Files as Metadata Following

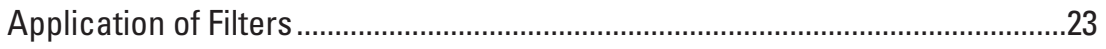

Option 3: Species Labels Are Provided in an Excel Spreadsheet ....................................24

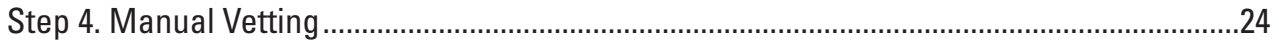

Mobile Transect Data Only ....................................................................................26

Download Results into an Excel Spreadsheet Format. ..............................................26

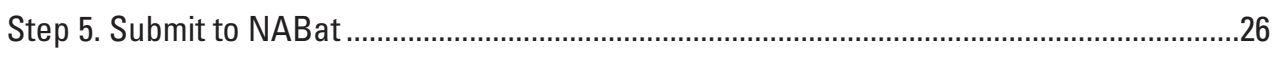

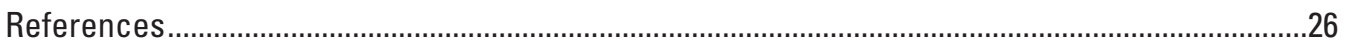

Appendix A. Case Study-Using Rule Sets to Process Acoustic Data for USDA

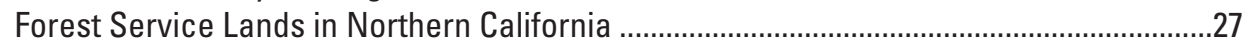

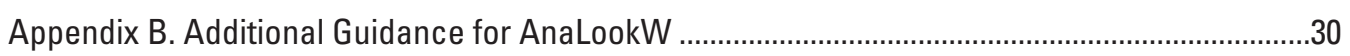

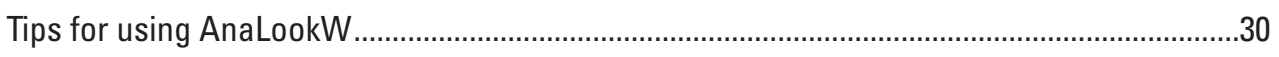

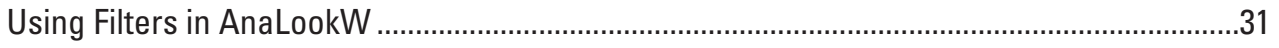

Appendix C. Bat Auto ID Species Confusion Matrix ................................................................... 


\section{Figures}

1. A general workflow for processing recorded acoustic files for the North American Bat Monitoring Program (NABat). Note: Workflow guidance varies between stationary point and mobile transect survey data at steps 2 and 4 , because objectives differ between survey methodologies (site occupancy from stationary point acoustic data versus relative abundance of species from mobile transect data) . .2

2. Vetting Table sorting criteria in SONOVET software …..................................................16

3. Startup user interface for EchoClass software (from Britzke, 2012) ..............................21

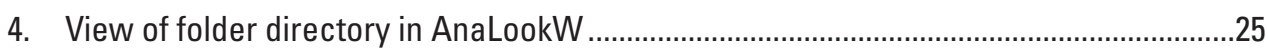

5. A standard output from SonoBat summarized in Excel (Szewczak and

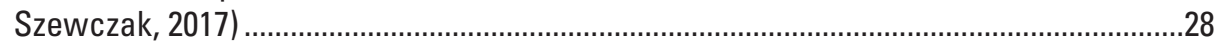

6. "Sort" criteria used to arrange summary output in Excel .............................................29

Tables

1. Required and recommended metadata fields for the North American Bat Monitoring Program (NABat) acoustic files. Metadata should be embedded in individual files. Fields with * should also be used for naming acoustic files .....

2. Suggested codes for acoustic detectors. Information provided by J. Tyburec (Bat Survey Solutions, LLC). 7

3. Species labels to be used when labeling acoustic files and submitting data to the North American Bat Monitoring Program (NABat) modified from Loeb and others (2015).

4. Species couplet, group, and frequency class labels recommended for labelling files with recordings of species that are acoustically similar and can occur sympatrically (adapted from Loeb and others, 2015). Also included are labels for files without bat recordings (NOTBAT) and for files that contain a bat pulse, but no grouping or user-defined category applies. While this is not an exhaustive list, we recommend you try to use these labels for consistency, but document any additional user-defined categories you use and submit to the North American Bat Monitoring Program (NABat). For consistency, always place the species names making up a couplet label in alphabetical order.

5. Example stationary GPS Locations for running Bat2012 in the above data structure ....20

6. Example sunset and sunrise times for running Bat2012 in the above data structure.....20 


\section{Conversion Factors}

U.S. customary units to International System of Units

\begin{tabular}{lll}
\hline \multicolumn{1}{c}{ Multiply } & By & \multicolumn{1}{c}{ To obtain } \\
\hline & Length & \\
\hline mile (mi) & 1.609 & kilometer $(\mathrm{km})$ \\
mile, nautical (nmi) & 1.852 & kilometer $(\mathrm{km})$ \\
yard (yd) & 0.9144 & meter $(\mathrm{m})$ \\
\hline
\end{tabular}

International System of Units to U.S. customary units

\begin{tabular}{|c|c|c|}
\hline Multiply & By & To obtain \\
\hline \multicolumn{3}{|c|}{ Length } \\
\hline kilometer (km) & 0.6214 & mile (mi) \\
\hline
\end{tabular}

\section{Abbreviations}

Auto ID automated species identification

BCID Bat Call Identification (software)

FFT Fast Fourier transform

FS Full spectrum recording format

GPS Global Positioning System

GRTS Generalized Random-Tessellation Stratified design

GUANO Grand Unified Acoustic Notation Ontology, a universal, extensible, open metadata format for acoustic files

KSPro Kaleidoscope Pro (software)

$\mathrm{ms} \quad$ millisecond $(1 \mathrm{~ms}=0.001$ seconds)

NABat North American Bat Monitoring Program

USDA U.S. Department of Agriculture

USGS U.S. Geological Survey

WAV Waveform audio-file format, standard for storing audio bit-stream on personal computers

ZC Zero-cross audio file format and recording format 


\title{
Guide to Processing Bat Acoustic Data for the North American Bat Monitoring Program (NABat)
}

\author{
By Brian Reichert, ${ }^{1}$ Cori Lausen, ${ }^{2}$ Susan Loeb, ${ }^{3}$ Ted Weller, ${ }^{3}$ Ryan Allen, ${ }^{4}$ Eric Britzke, ${ }^{5}$ Tara Hohoff, ${ }^{6}$ Jeremy \\ Siemers, ${ }^{7}$ Braden Burkholder, ${ }^{8}$ Carl Herzog, ${ }^{9}$ and Michelle Verant ${ }^{10}$
}

\section{Introduction}

The North American Bat Monitoring Program (NABat) is a multi-national, multiagency coordinated monitoring program designed to assess the status and trends of North American bats at local, state, and range-wide scales. NABat monitoring efforts focus on the 47 species of bats shared by Canada, the United States, and Mexico. NABat is composed of a network of partners including local, State, Federal, and provincial agencies, Tribes, non-governmental organizations, and volunteers collecting bat survey data and invested in bat conservation. Information from NABat can be used to inform land management and conservation decisionmaking.

The success of NABat is predicated on making reliable inferences about bat distributions and their relative abundance from data collected and processed using standardized techniques and protocols in a consistent manner over space and time. In 2015, "A Plan for the North American Bat Monitoring Program (NABat)" (Loeb and others, 2015) was developed, providing the foundation and overall direction needed for the initial implementation of NABat. Loeb and others (2015) represented the first step towards the establishment of standardized protocols, with the understanding that guidance would be refined and more specific protocols would be made available over time.

NABat relies in part on acoustic monitoring using stationary and mobile transect survey methodologies. Currently, however, acoustic data are being managed and processed using a variety of methods. These inconsistencies may introduce sources of undocumented, systematic bias that could limit the utility of these combined data for evaluating bat distributions and trends at broader scales. We believe there is a need for more specific guidance on detector settings, protocols for deploying acoustic detectors, and a standardized workflow for analyzing acoustic data. Standardized protocols and workflow can help to establish a common currency from which to improve the interpretation of acoustic data by reducing variation and uncertainty in how bat echolocation calls are collected, processed, and identified to species.

In 2017, the U.S. Geological Survey (USGS) Fort Collins Science Center hosted a workshop of bat acoustic experts with the goal of developing a suggested workflow to help standardize the analysis of acoustic data for NABat (NABat Acoustic Data Analysis Workflow Workshop, 2017). The guide that follows is a product of that workshop. While accurate and efficient analysis of bat acoustic data requires a minimum level of expertise and training, the suggested workflow is intended to help make this

\footnotetext{
${ }^{1}$ U.S. Geological Survey

${ }^{2}$ Wildlife Conservation Society Canada

${ }^{3}$ USDA Forest Service

${ }^{4}$ Bat Call Identification, Inc.

${ }^{5}$ U.S. Army Corps of Engineers

${ }^{6}$ Illinois Natural History Survey

${ }^{7}$ Colorado Natural Heritage Program

${ }^{8}$ Montana Natural Heritage Program

${ }^{9}$ New York State Department of Environmental Conservation

${ }^{10}$ National Park Service
} 
process more repeatable and feasible for a wider pool of potential NABat partners, including those with limited resources and experience. To this end, the workflow was developed with the intention of minimizing the amount of time and effort required for processing bat acoustic data for NABat while following a "conservative" approach that reduces subjectivity and minimizes the potential for species misidentification.

The following provides general recommendations and specific workflows for the process of identifying bat species from acoustic files recorded using both stationary point and mobile transect acoustic monitoring protocols for NABat (Loeb and others, 2015). It focuses exclusively on post-processing of acoustic data. For recommendations on detector settings and detector deployment, download the "NABat Guide to Site Selection for Stationary Point Monitoring" and the "NABat Guide to Acoustic Detector Settings" available on the "Resources" page of the NABat website (https://nabatmonitoring.org/\#/resources).

The described workflow includes data attribution (step 1), noise scrubbing (step 2), automated species identification (auto ID) (step 3), manual vetting (step 4), and submitting data to NABat (step 5, fig. 1). Some auto ID software automates the process and allows you to complete steps 1-3 at once. The workflow varies at step 2 and step 4 for stationary versus mobile transect monitoring because of the inherent differences between objectives (that is, species occupancy versus species relative abundance, respectively). For detailed workflows and tips for using specific software programs see chapters $1-5$ and appendixes A and B of this report.

This document is NOT meant to serve in place of the user manual or official documentation provided by the software developers. It should only serve to help users follow NABat protocols while using the available software. It is strongly recommended that users are familiar with the software user's manual before using this document.

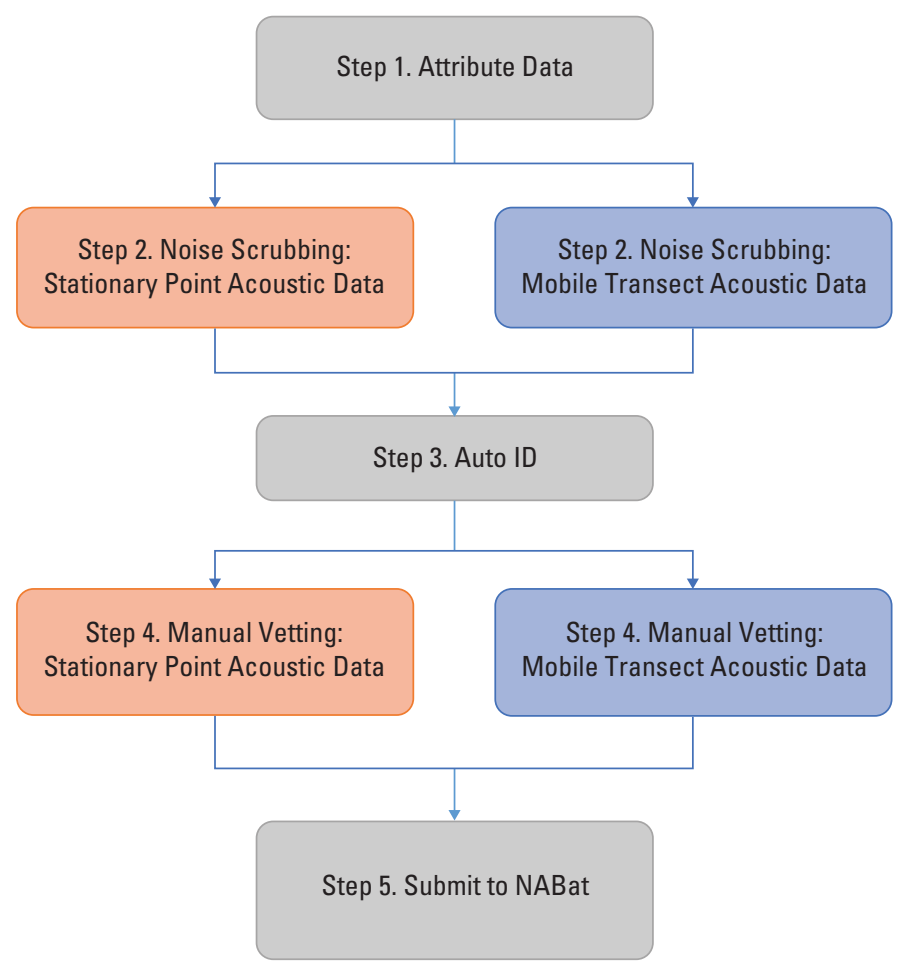

Figure 1. A general workflow for processing recorded acoustic files for the North American Bat Monitoring Program (NABat). Note: Workflow guidance varies between stationary point and mobile transect survey data at steps 2 and 4 , because objectives differ between survey methodologies (site occupancy from stationary point acoustic data versus relative abundance of species from mobile transect data). 


\section{Step 1. Attribute Acoustic Files}

Download cards or recorded acoustic files to a hard drive. Be sure to retain all status and log files associated with recordings (these are indispensable for trouble-shooting missing data and low data rates).

\section{Naming Acoustic Files}

Use Grid Cell GRTS ID, Site Name, and timestamp (using 24-hour clock notation) as a prefix for call file names (see table 1 for definitions). When put together, Grid Cell GRTS ID and Site Name provide a unique identifier for each detector deployment.

\section{Examples}

- 5867_NW_20160712_210932: This file was recorded in a NABat grid cell with GRTS ID 5867, at the NW site (or quadrant), on July 12, 2016 at 21 hours (or 9 pm) 9 minutes and 32 seconds.

- 5867_DT_20160712_214930: This file was recorded in a NABat grid cell with GRTS ID 5867, on a mobile driving transect (DT), on July 12, 2016 at 21 hours (or 9 pm) 49 minutes and 30 seconds.

To more easily compare surveys across grid cells and sites over time, maintain a consistent naming convention. For your reference, it can be helpful to develop an "authority file" of your naming conventions so (1) each code is unique and (2) additional description can be entered for each code to properly identify the site.

Depending on detector and software system used, this naming prefix can typically be pre-programmed prior to field recording, and (or) can be edited for entire batches of files during post-processing. For example, with recordings made with the Pettersson D500X ultrasound recording unit (Pettersson Elektronik AB, http://www.batsound.com/) Grid Cell GRTS ID and Site Name can be appended to timestamped files when files are scrubbed and attributed in SonoBat software v4.2.1 (Szewczak and Szewczak, 2017) using the D500x File Attributer utility or the DataWizard utility. Both utilities can be used on any waveform audio-file format (.wav) recordings, including situations where filenames cannot be altered within the detector or analysis software. For guidance on detector settings see the "NABat Guide to Acoustic Detector Settings," available for download here: https://nabatmonitoring.org/\#/resources.

\section{Assigning Metadata to Acoustic Files}

It is important that each recorded acoustic file also be attributed with metadata (that is, information about the recorded file). To meet the data management requirements for NABat, Grid Cell GRTS ID, Site Name, and Timestamp must also be included as metadata for each file (see table 1 for definitions). It is also strongly recommended that the information in table 1 be added as metadata to individual files to help contextualize recordings. This information summarizes the recording situation and should also be entered in the associated downloadable NABat Project Database (available for download at https://nabatmonitoring.org/\#/tools).

These metadata fields may be represented using the extensible Grand Unified Acoustic Notation Ontology (GUANO) metadata format as follows:

NABat|Grid Cell GRTS ID: 5867

NABat|Site Name: NW

Additional resources for attributing files with metadata will be downloadable at the NABat Project website (https://nabatmonitoring.org/\#/resources). See chapters 1-5 of this report for more details on how to assign metadata using specific software. 
Table 1. Required and recommended metadata fields for the North American Bat Monitoring Program (NABat) acoustic files. Metadata should be embedded in individual files. Fields with * should also be used for naming acoustic files.

[km, kilometer; WGS, World Geodetic System 1984]

\begin{tabular}{|c|c|c|}
\hline Field & $\begin{array}{l}\text { Required or } \\
\text { recommended }\end{array}$ & Description/Instruction \\
\hline *Timestamp & Required & Time and date when recording occurred. \\
\hline *Site Name & Required & $\begin{array}{l}\text { A user-defined name (6-character limit) of the specific location (or point) where a detector was } \\
\text { deployed within a single grid cell. Being consistent with site names from year to year allows } \\
\text { for easier sorting and interpretation of the data. For example, if four stationary detectors were } \\
\text { deployed, each within a } 5 \mathrm{~km} \times 5 \mathrm{~km} \text { quadrant in the same } 10 \mathrm{~km} \times 10 \mathrm{~km} \text { grid cell, Site } \\
\text { Names used to describe the four deployment locations could be 'NW', 'NE', 'SW,' and 'SE.' } \\
\text { Alternatively, Site Names can be based on a nearby town name, habitat type, property name, } \\
\text { and so forth. If a mobile acoustic transect survey was conducted within the same grid, an ad- } \\
\text { ditional unique Site Name, for example, 'DT' (mobile driving transect), would be applied. }\end{array}$ \\
\hline Site coordinates & Recommended & Latitude and longitude (WGS 84). \\
\hline Land unit code & Recommended & $\begin{array}{l}\text { A user-defined 4-letter abbreviation describing your study area, region, park, state, or land unit. For } \\
\text { example, if the survey is conducted in a park or refuge, use park or refuge codes (for example, } \\
\text { YELL for Yellowstone National Park). If the survey is not carried out in a park or refuge, the } \\
\text { user creates a 4-letter abbreviation for Land Unit Code based on the region or larger surround- } \\
\text { ing area (for example, SOCA for South Carolina, 3LMA for Three Lakes Wildlife Management } \\
\text { Area). Note that multiple sites (Site Names) can exist within the same land unit code. }\end{array}$ \\
\hline Contact information & Recommended & Name of person/entity that recorded the file. \\
\hline Detector type & Recommended & Brand and model \\
\hline Type of clutter & Recommended & Nearest clutter type (for example, vegetation, buildings, or other structure). \\
\hline Habitat type & Recommended & Broad habitat type surrounding microphone. \\
\hline Unusual occurrences & Recommended & $\begin{array}{l}\text { Indicate whether unusual occurrences took place during the recording session that may impact the } \\
\text { interpretation of results for example, power to detector may have been low, time not adjusted } \\
\text { for daylight savings time, recording was interrupted due to dead batteries, filled data card, } \\
\text { microphone or cable damage, schedule programming error, late deployment, deployment varies } \\
\text { due to non-standard microphone mounting, incorrect detector settings, and so forth.). }\end{array}$ \\
\hline Activation start time & Recommended & $\begin{array}{l}\text { Time when detector was activated to start recording. Note that this may be different than deploy- } \\
\text { ment start time. }\end{array}$ \\
\hline Activation end time & Recommended & Time when detector was deactivated. Note that this may be different than deployment end time. \\
\hline
\end{tabular}




\section{Step 2. Noise Scrubbing}

Acoustic sampling for bats results in the recording of both bat echolocation calls and extraneous noise (that is, non-bat files). In some circumstances, files with extraneous noise can represent a large proportion of the recorded files. The goal of noise scrubbing is to identify and separate files with only extraneous noise from files with bat echolocation calls. Noise scrubbing reduces storage requirements and increases efficiency of the call identification process.

Multiple automated methods can be used to remove extraneous noise. At its core, the noise scrubbing process represents an inherent balancing act between removing non-bat sounds and low quality bat echolocation calls. An optimized noise scrubbing process will result in retention of recordings of bats potentially identifiable to species while retaining a trivial number of non-bat recordings. Achieving this balance will depend on the software employed, the parameters specified and the recording situation. For example, in areas with high levels of background noise, it may be necessary to remove a larger proportion of low-quality echolocation recordings in order to minimize the number of files that do not contain bat echolocation recordings.

\section{Scrubbing Stationary versus Mobile Datasets}

For NABat stationary acoustic monitoring, where the goal is to document species presence based on identification of high-quality echolocation recordings, noise scrubbing specifications should be more stringent to retain mostly high quality calls. Whereas for NABat mobile acoustic transects, where the goal is to document the relative abundance of each bat species, noise scrubbing should be more lenient, where parameters are set to retain all potential bat recordings.

In general, the software packages described here provide robust methods for separating bat echolocation calls from non-bat, extraneous noise and are easily customizable to be more stringent or lenient in the files that pass. However, the various methods for automated noise filtering differ. Some programs have a separate file scrubber used as a standalone step (for example, SonoBat [chapter 2.] and SCAN'R [not presented here]) while other software programs integrate file scrubbing into the process of auto ID (that is, automated species identification, see step. 3 below, for example), Bat Call Identification, (BCID, chapter 3 and EchoClass, (chapter 4), and still others have it optionally as an integrated or standalone step (for example, Kaleidoscope, chapter 1). For zero-cross (ZC) files, scrubbing can also be done in AnaLookW v4.2g (Titley Scientific, 2016) (chapter 5.) using a filter prior to being analyzed in one of the auto ID programs. When starting with a new program, it may be helpful to review samples of stationary and mobile acoustic datasets post scrubbing to determine appropriate settings.

\section{Step 3. Auto ID}

The objective of using auto ID, or automated species identification, is to rapidly determine a species classification for large amounts of data in order to determine probable presence for the species of interest. Auto ID includes additional filtering to select pulses that are suitable for identification and then classify pulses and files. Classification methods vary among software programs but all rely on statistical comparisons of unknown pulses to classifiers or algorithms developed from a known call library of selected species. A critical part of this process is that the user must select the correct set of species to be used in this classification process. This is an important step as the user needs to make sure that all of the possible species in the state or region are included in the species set (including accounting for potential range expansions), yet excluding species that are unlikely to occur. Note that some software classifiers may have limited species support. Resources for determining potential species lists for each NABat grid cell are made available at https://api.sciencebase.gov/nabatmonitoring-reports/adHocCreator? catalogId=nabat\&repo rtId=projectReview\&columns=\%2Fprojects@prjctname,prjctlead,owningorg,grtscellid,grts_point,batspp, and via the "Map my Project" feature on on an NABat partner's project home page https://nabatmonitoring.org/\#/home/welcome.

Software programs for auto ID have varying levels of customization for species classification, including the degree of similarity required between known (underlying reference library) and unknown recordings for a species-level identification to be made by the program. In general, a more conservative (which hopefully leads to an increase in accuracy) level of classification is desired. These specifics are included in chapters $1-5$. Some programs embed species classification labels as metadata into the files of bat recordings (for example, SonoBat, Kaleidoscope Pro, BCID, and AnaLookW). Metadata formats are evolving; currently not all metadata written to a file by one program will be read by another program. Some software allows you to access and view files with auto ID metadata from within the software (SonoBat and Kaleidoscope Pro) while others require an additional software viewer be used if recordings are to be visualized (for example, AnaLookW can be used to view files that have been processed with BCID, EchoClass, or Kaleidoscope Pro). 


\section{Step 4. Manual Vetting}

Auto ID software programs are limited by various issues in the analysis process that can lead to inaccurate species classifications. Generally, programs are unable to account for deviations from expected call structure due to atypical bat behavior, approach calls, cluttered environments, presence of social calls, recorded call quality issues, multiple individual bats and (or) species in a recording, trends across a sequence of call pulses, and inherent overlap that exists in call metrics, pulse shape and (or) sequence pattern between some species. Both automated classification programs and manual vetting can also be limited by their underlying libraries of known calls. When there are biases in the reference library (for example, proportion of certain pulse shapes, under-representation of a particular species, and misidentification of recorded species), identification of unknown calls can also be inaccurate.

The goal of manual vetting acoustic files for NABat is to eliminate species misclassifications resulting from the automated identification process, because species misclassifications can lead to false-positive errors (that is, documenting that a species was present in a grid cell, when it was not). To meet this goal, manual vetting should focus on ensuring that auto ID classifiers have not misclassified files based on poor recording quality, non-search phase components of calls, recordings with multiple bats that may cause one or more bats to vary their echolocation outside the known norms for the species, or echolocation calls from species other than the species suggested from the auto ID. Manual vetting is a qualitative process that requires users with extensive experience.

\section{General Recommendations:}

- Accept the fact that not all call sequences can be, or should be, definitively identified to a species using diagnostic characteristics measurable in sound viewing/analysis software.

- View the recording in "real-time" (most viewers will default to showing recordings in "compressed view") to help visualize inter-pulse intervals (the relatively long periods of silence in between call pulses). Ensure that there is a consistent inter-pulse interval indicating that the bat was performing a typical "search-phase" call type and not performing a behavior such as an inspection or acquisition ("approach-phase" call type). Approach-phase behaviors result in call pulses with parameters that differ from search phase calls such as higher frequencies, broader bandwidth, and steeper slopes. Approach-phase pulses often show a high degree of overlap among species. To examine pulse shape parameters view in "compressed time."

- Limit the potential species list for a particular acoustic survey site by the potential geographic ranges of species; if a species is less likely to be present and does not have large overlap in call characteristics with more common species in the area, include it in the analysis. Otherwise, if a species is unlikely and has overlapping call characteristics with other species in the area, it is likely best to not include this species in the potential species list, until there is more evidence to justify looking for it acoustically.

- Review a single species at a time in order to be consistent and efficient in your manual review. This can be achieved in some programs (for example, Kaleidoscope Pro, SonoBat, BCID, and AnaLookW) by sorting species-labelled files (metadata embedded in files following automated ID) and then viewing files in order by species. If sorting of files is not available within the automated ID software, then an associated viewer software can be used (for example, BCID engages AnaLookW as a viewer following auto ID), or a results output file can simply be viewed in a tabular program or database (for example, Microsoft Excel file) where files can be sorted by species, and then viewed in a separate viewer (for example, ZC recordings analyzed in EchoClass can be manually viewed in AnaLookW).

- Review the entire sequence. Look for long call sequences, for example, those with multiple consecutive pulses in a sequence, especially pulses that have consistent inter-pulse intervals and consistent shapes, slopes, and bandwidths. The more calls with definitive features, the more confident the identification.

- You may want to examine recordings immediately before or after a file to determine if they provide any context in which to understand a particular pulse pattern (for example, if a bat has circled within range of the microphone, generating multiple files, a sequence of varying pulse shapes may better elucidate the species identification).

- Assess recordings that have a good "signal to noise" ratio (identified by crisp, clean, non-saturated oscillograms in fullspectrum recordings) and without non-bat noise, echoes, or distortions in the call-pulses that can skew the qualitative or quantitative measures of the individual call pulses.

- If available, use established regional keys or rulesets for acoustic species identification (for example, see appendix A. Case Study: Using Rule Sets to Process Acoustic Data for USDA Forest Service Lands in Northern California). 
- Files that are manually-vetted should be assigned one of the following: a standard 4 or 6-letter species label (table 3), species couplet (for example, MYCAMYYU, LANOTABR), frequency class (HighF, LowF), NoID, or NOTBAT (see table 4 for species couplet, group, and frequency class labels). Whether you choose 4- or 6-letter species labels, be consistent and avoid mixing labels for a single species. If it is potentially ambiguous as to whether an identification label in file metadata is from an auto ID or manual process, a small letter prefix is an accepted method to provide clarity. (For example, mANPA for a manually verified ANPA file to differentiate from an ANPA species label derived through other means that may also be listed in the metadata [for example filter or auto ID]; see AnalookW workflow chapter 5.). If prefixes or suffixes are used to denote different sources of identification labels, this should be clearly stated in the data entry into NABat.

Table 2. Suggested codes for acoustic detectors. Information provided by J. Tyburec (Bat Survey Solutions, LLC).

[Binary Acoustic Technology LLC, http://binaryacoustictech.com/; Pettersson Elektronik AB, http://batsound.com/; Titley Scientific, 2016; and Wildlife Acoustics, Inc., https://www.wildlifeacoustics.com/]

\begin{tabular}{|c|c|c|}
\hline Detector & Detector-Make/model/mic .type & Code \\
\hline BINARY ACOUSTIC & AR125 recording to PC/Mac using Spect'r & A125 \\
\hline BINARY ACOUSTIC & AR125-FG recording to PC/Mac using Spect'r & ARFG \\
\hline BINARY ACOUSTIC & AR180 recording to PC/Mac using Spect'r & A180 \\
\hline BINARY ACOUSTIC & iFR-V recording to on-board media & $\mathrm{iFRv}$ \\
\hline BINARY ACOUSTIC & AcroBat Mic Array & ABAR \\
\hline PETTERSSON & D1000x with external, cabled Mic & D1Kp \\
\hline PETTERSSON & D1000x with on-board Mic (for example, active recording/voucher) & $\mathrm{D} 1 \mathrm{Ka}$ \\
\hline PETTERSSON & $\mathrm{D} 240 \mathrm{x}$ recording direct to $\mathrm{PC} / \mathrm{Mac}$ & D240 \\
\hline PETTERSSON & D240x recording to iRiver/Zoom or other Digital Recorder & $\mathrm{D} 24 \mathrm{x}$ \\
\hline PETTERSSON & D500x with external, cabled Mic and cone off & D50o \\
\hline PETTERSSON & D500x with external, cabled Mic and cone on & D50d \\
\hline PETTERSSON & D500x with on-board Mic cone on (for example, active recording/voucher) & D500 \\
\hline PETTERSSON & M500 (USB mic) cone off; recording to PC/Mac/Tablet & M50o \\
\hline PETTERSSON & M500 (USB mic) cone on; recording to PC/Mac/Tablet & M50d \\
\hline TITLEY & AnaBat Express with external, cabled mic & ABex \\
\hline TITLEY & AnaBat Express with on-board mic & ABeo \\
\hline TITLEY & AnaBat SD1 with LoF mic & SD11 \\
\hline TITLEY & AnaBat SD1 with standard mic & SD1s \\
\hline TITLEY & AnaBat SD2 with LoF mic & SD21 \\
\hline TITLEY & AnaBat SD2 with standard mic & SD1s \\
\hline TITLEY & AnaBat Walkabout with directional accessory OFF & ABWo \\
\hline TITLEY & AnaBat Walkabout with directional accessory ON & ABWd \\
\hline WILDLIFE ACOUSTICS & EM-Touch with external, cabled EchoMeter Mic & EMTx \\
\hline WILDLIFE ACOUSTICS & EM-Touch with on-board EchoMeter Mic & EMTo \\
\hline WILDLIFE ACOUSTICS & EM-Touch2, regardless of extension cabling & EMT2 \\
\hline WILDLIFE ACOUSTICS & EM-TouchPRO, regardless of extension cabling & EMTP \\
\hline WILDLIFE ACOUSTICS & EM3/EM3+ with on-board mic & EM3o \\
\hline WILDLIFE ACOUSTICS & EM3/EM3+ with external mic & EM3x \\
\hline WILDLIFE ACOUSTICS & SM2Bat-192 with SMX-US Mic & SM2s \\
\hline WILDLIFE ACOUSTICS & SM2Bat-384 with SMX-US Mic & SM2s \\
\hline WILDLIFE ACOUSTICS & SM2Bat+ with SMX-US Mic & SM2s \\
\hline WILDLIFE ACOUSTICS & SM2Bat+ with SMX-UT Mic & SM2t \\
\hline WILDLIFE ACOUSTICS & SM2Bat+ with SMX-U1 Mic & SM2u \\
\hline WILDLIFE ACOUSTICS & SM3Bat with SMX-UT Mic & SM3t \\
\hline WILDLIFE ACOUSTICS & SM4BAT-FS with SMM-U1 Mic & SM4u \\
\hline
\end{tabular}


Table 3. Species labels to be used when labeling acoustic files and submitting data to the North American Bat Monitoring Program (NABat) modified from Loeb and others (2015).

[Kaleidoscope Pro uses 6-letter labels. Regardless of whether you choose 4- or 6-letter labels, be consistent and avoid mixing labels for a single species. Adding a prefix to lowercased letters on species codes helps to differentiate manually vetted labels, $\mathrm{m}$, from auto ID labels (for example, "mLANO" for label assigned to a file identified as Lasionycteris noctivagans through manual vetting)]

\begin{tabular}{|c|c|c|c|}
\hline Common name & Scientific name & 4-letter Label & 6-letter Label \\
\hline Pallid bat & Antrozous pallidus & ANPA & ANTPAL \\
\hline Jamaican fruit-eating bat & Artibeus jamaicensis & ARJA & ARTJAM \\
\hline Mexican long-tongued bat & Choeronycteris mexicana & CHME & CHOMEX \\
\hline Rafinesque's big-eared bat & Corynorhinus rafinesquii & CORA & CORRAF \\
\hline Townsend's big-eared bat & Corynorhinus townsendii & COTO & CORTO \\
\hline Big brown bat & Eptesicus fuscus & EPFU & EPTFUS \\
\hline Spotted bat & Euderma maculatum & EUMA & EUDMAC \\
\hline Florida bonneted bat & Eumops floridanus & EUFL & EUMFLO \\
\hline Greater bonneted bat & Eumops perotis & EUPE & EUMPER \\
\hline Underwood's bonneted bat & Eumops underwoodii & EUUN & EUMUND \\
\hline Allen's big-eared bat & Idionycteris phyllotis & IDPH & IDIPHY \\
\hline Silver-haired bat & Lasionycteris noctivagans & LANO & LASNOC \\
\hline Western red bat & Lasiurus blossevillii & LABL & LASBLO \\
\hline Eastern red bat & Lasiurus borealis & LABO & LASBOR \\
\hline Hoary bat & Lasiurus cinereus & LACI & LASCIN \\
\hline Southern yellow bat & Lasiurus ega & LAEG & LASEGA \\
\hline Northern yellow bat & Lasiurus intermedius & LAIN & LASINT \\
\hline Seminole bat & Lasiurus seminolus & LASE & LASSEM \\
\hline Western yellow bat & Lasiurus xanthinus & LAXA & LASXAN \\
\hline Mexican long-nosed bat & Leptonycteris nivalis & LENI & LEPNIV \\
\hline Lesser long-nosed bat & Leptonycteris yerbabuenae & LEYE & LEPYER \\
\hline California leaf-nosed bat & Macrotus californicus & MACA & MACCAL \\
\hline Pallas' mastiff bat & Molossus molossus & MOMO & MOLMOL \\
\hline Peter's ghost-faced bat & Mormoops megalophylla & MOME & MORMEG \\
\hline Southwestern myotis & Myotis auriculus & MYAR & MYOAUR \\
\hline Southeastern myotis & Myotis austroriparius & MYAU & MYOAUS \\
\hline California myotis & Myotis californicus & MYCA & MYOCAL \\
\hline Western small-footed myotis & Myotis ciliolabrum & MYCI & MYOCIL \\
\hline Long-eared myotis & Myotis evotis & MYEV & MYOEVO \\
\hline Gray myotis & Myotis grisescens & MYGR & MYOGRI \\
\hline Keen's myotis & Myotis keenii & MYKE & MYOKEE \\
\hline Eastern small-footed myotis & Myotis leibii & MYLE & MYOLEI \\
\hline Little brown myotis & Myotis lucifugus & MYLU & MYOLUC \\
\hline Arizona myotis & Myotis occultus & MYOC & MYOOCC \\
\hline Northern myotis & Myotis septentrionalis & MYSE & MYOSEP \\
\hline Indiana myotis & Myotis sodalis & MYSO & MYOSOD \\
\hline Fringed myotis & Myotis thysanodes & MYTH & MYOTHY \\
\hline Cave myotis & Myotis velifer & MYVE & MYOVEL \\
\hline Long-legged myotis & Myotis volans & MYVO & MYOVOL \\
\hline Yuma myotis & Myotis yumanensis & MYYU & MYOYUM \\
\hline Evening bat & Nycticeius humeralis & NYHU & NYCHUM \\
\hline Pocketed free-tailed bat & Nyctinomops femorosaccus & NYFE & NYCFEM \\
\hline Big free-tailed bat & Nyctinomops macrotis & NYMA & NYCMAC \\
\hline Canyon bat & Parastrellus hesperus & PAHE & PARHES \\
\hline Tri-colored bat & Perimyotis subflavus & PESU & PERSUB \\
\hline Brazilian free-tailed bat & Tadarida brasiliensis & TABR & TADBRA \\
\hline
\end{tabular}


Table 4. Species couplet, group, and frequency class labels recommended for labelling files with recordings of species that are acoustically similar and can occur sympatrically (adapted from Loeb and others, 2015). Also included are labels for files without bat recordings (NOTBAT) and for files that contain a bat pulse, but no grouping or user-defined category applies. While this is not an exhaustive list, we recommend you try to use these labels for consistency, but document any additional user-defined categories you use and submit to the North American Bat Monitoring Program (NABat). For consistency, always place the species names making up a couplet label in alphabetical order.

\begin{tabular}{|c|c|c|}
\hline Common name & Scientific name & Label \\
\hline Pallid bat, Big brown bat & Antrozous pallidus, Eptesicus fuscus & ANPAEPFU \\
\hline Big brown bat, Silver-haired bat & Eptesicus fuscus, Lasionycteris noctivagans & EPFULANO \\
\hline Western red bat, Canyon bat & Lasiurus blossevillii, Parastrellus hesperus & LABLPAHE \\
\hline Eastern red bat, Tri-colored bat & Lasiurus borealis, Perimyotis subflavus & LABOPESU \\
\hline Eastern red bat, Little brown bat & Lasiurus borealis, Myotis lucifugus & LABOMYLU \\
\hline Eastern red bat, Seminole bat & Lasiurus borealis, Lasiurus seminolus & LABOLASE \\
\hline Silver-haired bat, Mexican free-tailed bat & Lasiurus noctivagans, Tadarida brasiliensis & LANOTABR \\
\hline Hoary bat, Mexican free-tailed bat & Lasiurus cinereus, Tadarida brasiliensis & LACITABR \\
\hline Long-eared or Keen's myotis, and Northern bat & Myotis evotis, Myotis keenii, Myotis septentrionalis & LEMY \\
\hline California bat, Yuma bat & Myotis californicus, Myotis yumanensis & MYCAMYYU \\
\hline Not a bat & & NOTBAT \\
\hline \multicolumn{2}{|l|}{ Bat, but no grouping or user-defined category applies } & NoID \\
\hline & User-defined categories & \\
\hline User-defined frequency class & $\begin{array}{l}\text { Various species with pulses that have a minimum frequency } \\
\text { of approximately } 15-25 \mathrm{kHz} \text {. }\end{array}$ & $25 \mathrm{k}$ \\
\hline User-defined frequency class & $\begin{array}{l}\text { Various species with pulses that have a minimum frequency } \\
\text { in the range of } 35-45 \mathrm{kHz} \text {. }\end{array}$ & $40 \mathrm{k}$ \\
\hline User-defined frequency class & $\begin{array}{l}\text { Various species of Myotis with pulses that have a minimum } \\
\text { frequency in the range of } 35-40 \mathrm{kHz} \text {. }\end{array}$ & 40kMyo \\
\hline User-defined frequency class & $\begin{array}{l}\text { Various species with pulses having a minimum frequency } \\
\text { lower than } \sim 30 \mathrm{kHz} \text {. }\end{array}$ & LowF \\
\hline User-defined frequency class & $\begin{array}{l}\text { Various species with pulses having a minimum frequency } \\
\text { higher than } \sim 30 \mathrm{kHz} \text {. }\end{array}$ & HighF \\
\hline
\end{tabular}

\section{For Stationary Point Acoustic Data}

- Because the goal is to determine site occupancy for each species, examine and manually ID at least one high quality, confidently identified recording per species per detector per night (that is, a voucher file) that was identified using an acoustic ID program. Where possible, select noise-free, long duration call sequences that can be confidently distinguished from conspecifics.

- High quality recordings generally result from a loud signal as a bat approaches closer to a microphone. Some potential indicators of higher quality recordings for some species (for example, Myotis spp.) include high bandwidth calls. High bandwidth indicates that the bat has approached the microphone close enough for high frequencies to be recorded. Close approach of bats can also be assumed in the presence of harmonics (in full-spectrum recordings), but note that some calls may be oversaturated if the bat approached the microphone too closely (as seen in clipped, or "squared off," oscillograms and the presence of aliasing, instead of true harmonics); sequences with oversaturated pulses should not be considered in the analysis process. 
- When corroborating automated species identification, focus your review on low clutter, search phase calls, and take precautions to avoid using approach phase calls, feeding buzzes, and social calls for most species.

- High quality recordings for auto ID have consistent inter-pulse intervals indicative of typical search-phase behavior. Be careful not to include approach phase calls when a bat is responding to an object in its flight path, as these calls take on a different shape that is generally less desirable for differentiating species, and can confound auto ID.

- Recordings should have been made in relatively uncluttered environments, however, low clutter calls can be difficult to obtain. For example, even when detectors are placed far from buildings, dense vegetation, or other hard surfaces bats often produce high clutter sequences when approaching the ground or in close proximity to other bats or while checking out the microphone if it is a novel item of interest on the landscape.

- Look at the entire sequence in both "compressed" and "real time" views. This will help you see the whole picture (for example, multiple bats and or species in the recording or non-search phase calls, indicating the types of convergent approach-phase and acquisition-phase call types that all bats are capable of producing).

- Adjust filters, thresholds, and other viewer settings as needed.

- If possible, play the recording in real-time or time-expansion to listen for a consistent cadence between call pulses.

- Examining files within a short period of time (for example less than 2 seconds) immediately prior and following the file of interest, may add context to better identify the species.

- To expedite review, focus manual vetting efforts on call sequences that were assigned an automated species identification. These files are more likely to contain high quality call sequences, unlike the files that did not receive an automated species identification. Spreadsheet outputs can be sorted in order of auto ID confidence by looking at the number of call pulses that contributed to the identification. In this way the longest call sequences and decisions most likely to be correct are presented first.

- Review auto ID files that have been assigned to a given species category. Confirm species identification for a minimum of one file per species per night (voucher file).

- For example if an auto ID assigns a file a label of Species $X$ because all 40 pulses in the sequence are assigned to that species, then this would be your voucher file for that species that night. If it is assigned to Species $X$ but only 20 of 40 pulses matched to this species label, and the other 20 assigned to Species $Y$ for which you think the sequence could equally be either species due to a lack of any diagnostic features, then you might consider a species label $X Y$. Then continue to review other files that were strongly assigned the Species $X$ label until one is found that represents a good voucher file. If a species' identification cannot be manually confirmed by examining the files with the highest auto ID confidence scores, you may also want to examine high quality recordings that were not assigned a species-specific auto ID label (for example, "NoID”). This is because sometimes the presence of more than one bat in a recording can confound auto-identification, despite a high quality recording.

\section{For Mobile Transect Acoustic Data}

- The goal for collecting mobile transect acoustic data for NABat is to evaluate relative species abundance. Therefore, manually vet all files, focusing first on the files that were assigned an automated species identification, as these are likely to be of higher quality. Sort the files by species and use the same criteria as described above for stationary point acoustic datasets.

- For files that did not receive an auto ID (that is, file-level classification results were left blank, or labeled HighF, LowF or NoID), if possible, apply filters or a sort function to enable like recordings to be viewed in series. For example, some auto ID software provides a suggestion of species pulse shapes that may be present in a file even if an auto ID could not be achieved. Alternatively, use the general call shape and characteristic frequency to sort "No ID" files by phonic group so that you have fewer species to consider at one time in the manual vetting process. For example, all low frequency bats may be viewed in series before all high frequency bats.

- When appropriate, assign a label to "No ID" files, preferentially using couplets, or categories that contain only two species (for example, LABOMYLU for pulses that have minimum frequencies $\sim 40$ kilohertz (kHz) but undulate up and down to some extent); recordings that could be of more than two possible species will receive a generic group category such as "Low Frequency bat" (for example, LowF or 25k Hz). 
- Because the goal of transects is to determine relative species abundance, it is also important to identify recordings that contain more than one individual bat and (or) species. Be sure to assign multiple labels as appropriate.

\section{The Manual Vetting Process}

\section{Assess call sequence quality.}

There are cases when auto ID is questionable due to reduced call quality. The presence of the following quality issues likely impact the effectiveness of auto ID software. Downgrade the species identification to a species group if one or more of the following issues exist.

- Excessive noise.

- Classifier chose inappropriate calls (pulses) for classification (for example social calls, quiet/out of range calls), included noise as part, or in place of a call, or used non-search phase or high clutter calls.

- Identification is confounded by multiple species or individuals present in the call sequence.

- Insufficient number of pulses present for confident identification.

- Destructive interference caused by echoes.

2. Examine call sequence and call characteristics.

If the auto ID is correct, the basic measures of the calls and sequence (for example characteristic frequency, duration, slopes, call intervals, and so forth) should be within the typical ranges observed for that species. Sometimes these call characteristics are measured incorrectly by the auto ID software, leading to assignment of a pulse sequence of an unlikely species. When the call characteristics are not within the range typically observed for the species identified, downgrade the species identification to a species group.

\section{Look for evidence of alternative species identification.}

Many call sequences have characteristics that fall within the ranges of multiple species. Depending on the potential species present, look for any evidence that the recording is actually of another species. Appendix C provides a "Bat Auto ID Species Confusion Matrix" as a quick look-up guide indicating which species are commonly confused by auto ID software. If there is strong evidence of another species, or a high degree of ambiguity (for example, 50 percent of the pulses match Species $X$, and 50 percent match a highly similar Species $Y$ ), downgrade the species identification from the auto ID to a species group " $X Y$ " (for example, EPFULANO). If there is no evidence of another species, and the recording is of a high-quality bat pass, accept the auto ID as correct for that file. However, auto ID software is currently evolving, and there are known cases of areas where one species might repeatedly be called another species. There are many reasons why this might be the case, such as regional differences in some species echolocation repertoires that may not be reflected in underlying libraries or unreliable known calls in a program's library. If the expert analyst can provide suitable justification for changing a species identification from auto ID to another species label, then such justification can be provided in an appended note, such as in the file metadata.

4. Look for evidence of multiple individuals in the file.

Depending on how the detector was deployed in relation to surrounding habitat features, flight paths, and so forth, passive recordings (from stationary point surveys) can include multiple individuals in a file. This is especially true if the microphone is located near a potential roost. Sometimes you can identify new roosts, or night roosts, acoustically by such recordings, so these instances are important to document. For example, in a passive dataset, a note in the metadata might be used to indicate there are many bats, or a suspected/known roost, and so forth. This should also be included in the submission of covariate information to NABat.

If multiple bats occur in a single recording from a mobile driving transect, it is important to tally the number of individuals. This is best done by including two labels for each file. Using software that enables metadata to be stored within the file, there should be one label per individual, even if the label is the same. For example, in Kaleidoscope, AnaLookW, or SonoBat the file "5867_NW_20160712_210932" containing two bats, each identified as big brown bat or silver-haired bat, would have the following species labels: EPFULANO,EPFULANO, where a the second species label is manually entered and preceded by a comma with no spaces. Note that some software packages tally labels for you (for example, "Countlabels" tool in AnaLookW would provide a tally and show "2 EPFULANO" for the file 5867_NW_20160712_210932 in the above example), however, this is not the desired format for NABat data entry. 


\section{Step 5. Submit to NABat}

The following steps are required for submitting acoustic monitoring data to NABat.

1. Request an Account on the NABat website by visiting https://nabatmonitoring.org/\#/home/welcome and clicking on "Request an Account". Once you have created a user profile and password you, must log in to gain access to your home page "My NABat Projects" with a list of your NABat projects.

2. Create a New NABat Project by visiting https://nabatmonitoring.org/\#/home/welcome, logging in, and clicking on "My NABat Projects."

A. A list of current projects will appear (for new users this list will be blank unless another Project Leader has added you to an existing NABat Project.) Click "Add New Project."

B. Enter required project information including Project Name, Owning Organization, and Project Description. Identify your role in the project. If you would like to allow other existing NABat users (that is, those with registered accounts on https://nabatmonitoring.org/\#/home/welcome) to access your NABat, click "Add User" and identify their role in the project.

C. If all or some of your projects follow the NABat priority sample design (that is, selecting grid cells following GRTS order), click "Cell Selection Tool" and follow the tool instructions to identify your NABat grid cells. Remember to provide reasons for not selecting potential grid cells in your study area or jurisdiction.

D. If your survey locations (for example, caves, mines, stationary acoustic point locations, mobile acoustic routes) were established prior to NABat (legacy data) or selected without consideration of the NABat sample design (found data), answer 'yes' to this sample design question and enter grid cell numbers manually. Also, indicate which survey types were conducted in each of the grid cells.

E. Click "Save Project."

3. View Project page by clicking on the name of your project on your home page (login is required).

4. Download the NABat Project Database (Microsoft Access, .accdb file) specific to your project by clicking "Download NABat Project Database". It is important that you DO NOT ALTER THE NAME OF THIS FILE. Each NABat Project is assigned a unique Project Key (see example above), which is automatically generated upon project creation and used in the naming of your NABat Project Database file. Altering the file name of your NABat Project Database will make it difficult to ensure that your database is linked to your project upon submission. The NABat Project Database can be used to enter data for all five NABat survey types (that is, Stationary Point Acoustic Surveys, Mobile Transect Acoustic Surveys, Internal Winter Hibernaculum Surveys, Internal Summer Maternity Colony Surveys, and Emergence Count Surveys). For acoustic surveys, users will need to provide the names and versions of software used for species identification. For directions on how to use the NABat Project Database download the "NABat Project Database User Manual" from https://nabatmonitoring.org/\#/tools. Currently, using the NABat Project Database is recommended for submitting your data to NABat, however, additional pathways for data submission will become available on your Project page.

5. Submit data to NABat by clicking on "Choose File" on your project page. Once acoustic files have been processed (that is, bat echolocation data have been identified to species following guidance in this document) and data are entered into your NABat Project Database, upload the following three items.

A. Summary table of acoustic files: This table is provided as output by auto ID software (.xlsx or .csv file formats are acceptable) and should include a column indicating which acoustic files were manually-vetted (see guidance below for details).

\section{B. NABat Project Database}

C. Acoustic files (.wav, wac, and (or) .zc file formats). See step 1 (Attribute Acoustic Files section of this document) for guidance on naming your acoustic files. Note that the ability to upload acoustic files will depend on the speed of your internet connection and may not be possible if your connections is slow. For this reason, please prioritize submission of "voucher files" (that is, acoustic files that have been manually vetted). For stationary point acoustic data, a voucher file is a single file providing evidence that a species was present at a single detector, within a single night. For mobile transect acoustic data, every file is considered a voucher and should be uploaded. If uploading acoustic files via the internet is not possible, contact Brian Reichert at breichert@usgs.gov for mailing instructions. 


\title{
Chapter 1. Processing Acoustic Data Using Kaleidoscope Pro Version 4.3.2
}

\author{
By Cori Lausen² and Susan Loeb ${ }^{3}$
}

${ }^{2}$ Wildlife Conservation Society Canada

${ }^{3}$ USDA Forest Service

\section{Step 1. Attribute Files}

- Ensure that minimum attribute data are included for each file. (See step 1, Attribute Acoustic Files that follows the Introduction of this report for more detail on attribution data).

- If input is in Anabat ZC files (Titley Scientific v.4.2g, 2016), attribute files with metadata in AnaLookW using the Global Header Change (see AnaLookW Workflow), or in Batch Tab of Kaleidoscope Pro (KSPro) (see the next step).

- If input is full spectrum (FS) files (for example, .wav file format), attribute files with metadata in the Batch Tab (by selecting "Prepend notes to output medadata") in the dropdown menu on the left side of the tab (that is, "INPUTS" section). Note: Metadata formats are currently evolving toward a unified system called "GUANO" which will be readable across all software programs that recognize GUANO metadata (for example, SonoBat). The process for entering metadata into GUANO will change in future versions of KSPro. In its current version (version 4.3.2), enter metadata in GUANO format by typing the following information into the end of the notes field.

GUANO|Version: 1.0

NABat|Grid Cell GRTS ID: 54405

NABat|Site Name: SW

These metadata values must each be on separate lines, and the fieldnames are spelling and case sensitive. In the example above, 54405 is the GRTS ID of your grid cell, and the detector was deployed in the NW quadrant of the grid cell.

Continue through steps 2 and 3 before processing files.

\section{Steps 2 and 3. Noise Scrubbing and Auto ID}

1. On Batch Tab, enable noise filtering — either delete or move to noise folder

2. On Batch Tab select input and output directories for each of the INPUT and OUTPUT sections. To do this use the "Browse" buttons. The OUTPUT folder must not be embedded within the INPUT folder or you will receive an error message.

3. Choose output file type. Click either WAV files or ZC files as appropriate.

- If input is WAV files and you plan to review files in Kaleidoscope or other full spectrum recording format (FS) viewer, click WAV as the output file type.

- If input is WAV file and you plan to review in AnaLookW or further analyze in Echoclass or BCID, click ZC as the output file type.

- If input is ZC file, click ZC as the output file type.

- Be sure to check off the box to include subdirectories if the input data consists of subfolders.

- Optional (but highly recommended if your files are not already sorted into "night" folders): Under the Create Subdirectories option choose "Nightly" which will sort the new files into nightly subfolders (based on "bat nights" with the date not changing at midnight). Ignore this step if your files are already in "night" folders.

4. Go to Signal Parameters Tab.

- Minimum signal of interest should be appropriate for the expected bat community. 
- Call duration should be one to 50 milliseconds (ms) for mobile transects and 2-50 ms for stationary points. This will ensure that all potential bat recordings are included in the mobile transect dataset (some noise files that do not contain bats will also be included) and will potentially exclude a few bat recordings in stationary datasets but will ensure more high-quality recordings for species identification. This is to ensure no bat files are missed in the measuring of relative abundance in the mobile transect dataset, even though it is anticipated that more of the auto-identified files will remain as NoID or changed in manual vetting, such as downgrading to a species group or frequency class.

- Minimum number of pulses should be three.

- Leave the maximum inter-syllable gap at its default value of $500 \mathrm{~ms}$. This applies only to the cluster analysis process, which is not part of this workflow.

- If the workflow remains within Kaleidoscope-Pro, check the Enhanced Signaling Processing box; otherwise do not check this box.

5. Go to Auto ID for Bats tab.

- Select appropriate species for your area. Make sure to consider species that may be rare or slightly out of range.

- Select "More Accurate-Conservative" option

6. When all settings are entered, press "Process Files" button.

\section{Step 4. Manual Vetting}

1. In the results window, sort by Folder, Matching, Date, and then by Auto ID. If some of these column names do not appear for you, select "File" => "Edit Columns" to show them. Review "Match Ratio" to confirm that this is a potentially good file, (that is, the more calls in a sequence that match the auto ID species, the better).

2. Look at the potentially good files (high matching values) to confirm they are the species identified. You may need to adjust the viewer settings, including viewing of ZC or FS pulses, FFT settings, brightness, and contrast, to best match your recordings.

3. If your manual verification agrees with the Auto ID, put the species name in the "Identification" field (this will be the same as the Auto ID, just mouse click on the Auto ID button below the "Identification" field). This will indicate to NABat that the file has been examined and been verified. There is no need to look at more than a few files if there is agreement that this species was present at a detector during the night in question.

4. If your manual verification does not agree with the Auto ID, put the revised species group label (or species if justification can be provided) in the Identification field.

5. Remember that you can enter more than one species label into the Identification field. If you see another bat species present, you can add this species label in by manually typing the label after a comma (do not use any spaces). For example, MYOLUC,LASCIN. (This process is automated by holding the control key during labelling in later KSPro versions.)

6. Save your results file.

\section{Step 5. Submit to NABat}

See the Introduction of this report for the steps for submitting data to NABat. To easily identify voucher files that should be prioritized for upload through your NABat Project page using KSPro, sort, select and save manually-vetted files. 


\title{
Chapter 2. Processing Full Spectrum Acoustic Data Using SonoBat 4.2.1
}

\author{
By Ted Weller ${ }^{3}$
}

${ }^{3}$ USDA Forest Service

\section{Step 1. Attribute Files Using SonoBat Attributer}

Ensure that minimum attribute data are included for each file. (See step 1 in the Introduction of this report for more detail on attribution data). SonoBat Batch Attributor attributes recordings and (optional) simultaneously scrubs (identifies and removes) noise files.

\section{Step 2. Noise Scrubbing using SonoBat Scrubber and Attributer}

Follow these steps to remove non-bat files from dataset.

1. Output to a new directory (for example, SiteDate_scrubbed) if retention of files identified as non-bats is desired.

2. Select "Medium Scrub" option.

3. For sites with low frequency bats (less than $[<] 20 \mathrm{kHz}$ ), choose to include signals from $5-20 \mathrm{kHz}$. Low frequency bats can include Euderma maculatum, Eumops spp., Lasiurus cinereus and Nyctinomops spp. Generally, consider including all $5-20 \mathrm{kHz}$ because it is often likely that most locations will have at least one low frequency bat species. However, for sites outside the suspected range of low frequency species, choose "exclude all signals $<20 \mathrm{kHz}$."

\section{Step 3. Auto ID}

Follow these steps to SONOBATCH the entire directory containing the recording session of interest.

Settings: If files up to $15 \mathrm{~s}$ long have been recorded, as recommend in Loeb and others (2015), ensure Preferences $=>$ Max Segment to Process reflects this file length.

1. Select the classifier most appropriate for your location and recording conditions.

2. Use "autofilter" generally.

3. Use "autofilter-low" in areas with low-frequency bats present (Euderma maculatum).

4. Use the default settings.

5. Acceptable call quality $=0.80$

6. $\quad$ Sequence Decision Threshold $=0.90$

7. Set Maximum Number of Calls to Consider per File $=16$

8. (OPTIONAL) To work with output in Excel, use "Output Legacy SonoBat Sheets" and select the "Classify?" option. Alternatively, if manual vetting in SONOVET skip to next step.

\section{Step 4. Manual Vetting}

Manually vet the SONOBATCH output depending on objectives (stationary point vs mobile transect acoustic monitoring). 


\section{Stationary Point and Mobile Transect Data}

Follow these steps to confirm species detections per detector per night.

1. Create Project in SONOVET by adding directory containing the SONOBATCH call files.

2. In the Vetting Table add a Monitoring Night Column by right-clicking on the header bar and Insert before $=>$ SonoBat Data $=>$ MonitoringNight.

3. In the Settings tab, sort the Vetting Table as follows. This sorts the results by night and species with the files with the highest probability of being confirmed by manual vetting at the top.

Note: One can use "Save Layout" to quickly apply these settings to future SONOVET projects.

\begin{tabular}{l|l|l|l|} 
MonitoringNight & A-z & & Z \\
\hline 1st & A-z & & Z \\
\hline \#Maj & Z-a & & Z \\
\hline \#Accp & Z-a & & $\mathbf{Z}$ \\
\hline 2nd & A-z & & $\mathbf{Z}$ \\
\hline Fc mean & A-z & & $\mathbf{Z}$ \\
\hline Dur mean & A-z & & $\mathbf{Z}$ \\
\hline
\end{tabular}

Figure 2. Vetting Table sorting criteria in SONOVET software.

4. For each monitoring night, confirm species ID for one file per species per night. Keep reviewing auto ID files where "SppAcc" = species $X$ until you confirm the species, or until you have reviewed what you would consider a reasonable number of files (for example, if the species cannot be confirmed after review of the 10 "most likely" files, you might assume non-detection for that species on that night.)

5. Files are displayed in SonoBat by clicking on the file row.

6. For each file reviewed, select a standard identification code in the Species Manual ID column by right clicking the Species Manual ID cell.

7. A standard code can be a 4-letter species code, a species couplet (for example, MYCAMYYU, LACITABR), a frequency group (for example, Q40k, Q25k) or a high/low frequency class (HighF, LowF).

8. Add custom species couplets and frequency groups using Config Manual ID Defaults.

9. Assigning files to groups helps track whether files have been reviewed.

\section{Mobile Transect Data Only}

The objective is to confirm species detections for all other files. Follow steps as above, but continue vetting all files, even those that have not been assigned an auto ID. This process is made easier by sorting the table by files that are likely to belong to the same phonic group so they can be viewed in series.

\section{Step 5. Submit to NABat}

See the Introduction of this report for the steps for submitting data to NABat. To easily identify voucher files that should be prioritized for upload through your NABat Project page in SonoBat, sort, select and save manually-vetted files. 


\title{
Chapter 3. Processing Acoustic Data Using Bat Call Identification ver. 2.7d (BCID)
}

\author{
By Ryan Allen ${ }^{10}$ \\ ${ }^{10}$ Bat Call Identification, Inc.
}

\section{Step 1. Attribute files}

In BCID (Bat Call Identification, v2.7d, 2015), data can be attributed in two ways - both have advantages and disadvantages.

1. Use Header tab in BCID-Anything that you type in this area will appear in the header of the ZC file for any file in your tree that passes the filter. The advantages are you can set the filter to look for something very specific and label files as such and can label an entire file tree at once. The disadvantage is that files that do not pass the filter (that is, noise files, poor quality calls) will not be labeled. (However, this can save a lot of processing time.)

2. Use Global Header Change in AnaLookW-You can change the headers of the file using the global header change options in AnaLookW. Make sure you "MARK" all files first, as changes will not take place on unmarked files (see chapter 5). This method will allow you to change all the files including noise files, but may be more time consuming.

All global positioning system (GPS) data should be entered into files using AnaHead located within AnaLookW at this time. BCID will work to include this in the near future making it easier on the user.

Data files can be arranged in any manner the user sees fit as long as all of the files to be analyzed are nested in the file directory selected to analyze the current "chosen" folder. We recommend a format of Grid Cell/Detector/Date for NABat.

\section{Step 2. Noise Scrubbing}

While the noise filtering process in BCID is relatively integrated there are some options that the user should be aware of. First, the user can change any of the parameters in the noise filter that they wish, but if this is done it should be done in a manner that is highly thought out. In addition, BCID recommends reporting significant changes in a header data type format. The filter that was used can be read on the last tab of the BCID output file.

The most important items that users can adjust within the filter are the Minimum Number of Calls to be considered in order to make an identification and the Min DP (Minimum Discriminant Probability). Increasing both or either of these numbers will have an effect on both false and true positives. For NABat use a minimum of three calls.

Given the guidance in this document, BCID would recommend experimenting with these numbers, especially the Min DP as increasing it from the default of 0.00 can help reduce false positives because the number is based on several values that deal with call quality.

If you would like to view the files as the software does in order to test your filter, you can create the filter under the filter tab within the BCID software and then load the filter into AnaLookW and view your files. This can also be useful for moving your "noise" files into separate folders.

\section{Step 3. Auto ID}

If you are using full spectrum files you must first run them through the wav to zero cross converter within the BCID software or other similar software packages.

The most important thing you can do is to choose the correct species for your area. Under the species tab in BCID you can choose a state or territory and then all of the species known to occur within that region will appear. Those that are widespread will be checked, those that are geographically isolated will be left unchecked. Those that are greyed out are not available for identification in the software.

We recommend setting the Create ANL file under the Advanced tab. This will allow the user to view the files in the next stage (manual vetting) by species label in AnaLookW. In addition, there is the option of adding the predicted species label into the header of the file by checking the corresponding box.

The final step is to use the Add Folders to Project button under the Project tab. You will select the folder at the highest level you would like your files analyzed. All files within and below the chosen folder will be included in the analysis and any labeling, 
etc. that you have chosen for the software to perform. Once all files are chosen, return to Project tab and click Analyze files. This will produce a .txt document will all the files, IDs, etc. and a Microsoft Excel file with three tabs: (1) all of the files broken down by folders, (2) summaries of all folders, and (3) settings used, including the filter parameters and species selected.

\section{Step 4. Manual Vetting}

The easiest method of manual vetting files in BCID is to use the ANL file that can be created by BCID under the "Advanced" tab and opened in AnaLookW. This will allow you to open a virtual folder of all files that BCID has classified to species and quickly review them to see if any of the files are consistent with calls produced by that particular species. Adjust header labels following guidance for manual vetting described above.

After manually vetting a dataset, one could re-run the auto ID, testing various values of the Min DP setting to determine the Min DP value that produces the results most similar to your manual vetting for a particular site, geographic region, etc. This could be informative for future analyses of datasets from this site. It is important to understand that both the software and manual vetting can be incorrect on occasion. The goal is to find the number where one can achieve the largest volume of data while ensuring the accuracy of the results. Once this number is arrived at, the software can be run using this number and the robustness of the results should be much more reliable.

\section{Step 5. Submit to NABat}

See the Introduction of this report for the steps for submitting data to NABat. To easily identify voucher files that should be prioritized for upload through your NABat Project page in BCID, sort, select, and save manually-vetted files. 


\section{Chapter 4. Processing Zero-Cross Acoustic Data Using EchoClass v 3.1}

\section{By Eric Britzke}

${ }^{4}$ U.S. Army Corps of Engineers

The following describes a recommended workflow using EchoClass for processing acoustic data for the North American Bat Monitoring Program (NABat) where data were collected or converted to zero-cross files $\left({ }^{*} .00 \#\right.$ file format).

\section{Step 1. Attribute files}

The first step in this process is attaching metadata to the files. The current version of EchoClass relies on the directory structure to provide information on the files instead of adding this metadata to the individual files (Britzke, 2012). Thus, in order to attach metadata to the individual files, use process included in chapter 5 (Recommended Workflow for Processing Zero-Cross Acoustic Data using AnaLookW).

\section{Tips for organizing your data}

The following information is taken from Britzke (2012). The data file structure must have at least two levels (no maximum) of folders for the program to run. Additionally, the structure must be consistent throughout for each run of the software. Keep the folder names short and do not include the "\&" symbol in any folders or filenames. All of the recorded call files must be in a folder named with 8 numeric characters (for example, 20120805). This can either be the date that the files were recorded or can be a single download data as EchoClass uses this information to look for files but reads date data from the individual file. As the output of the analysis software includes information from the directory structure, (following the example below) the directory structure will populate the output with project and site information that is then ready for inclusion in a report.

\section{Example file structure}

\section{Bats2012}

Project1

Grid Cell1

Site 1

20120601

20120602

Site2

20120701

- If you select the folder 20120601 the output file will be empty.

- If you select the folder Site1 you would get results from all folders named with 8 numeric characters under the Site1 folder (for example, 20120601 and 20120602).

- If you select the folder Project1 you will get results from all of the folders named with 8 numeric characters in all of the sites under the Project1 folder (for example, 20120601, 20120602, and 20120701).

- If you select the folder Bats2012 you will get results from all of the folders named with 8 numeric characters in all of the sites of all of the projects under the Bats2012 folder. 


\section{Global Positioning System (GPS) Information}

\section{Mobile Transect Acoustic Monitoring}

- Make sure the gps.txt file downloaded with CFC read is located within each date folder.

- GPS_and_Sunset_Master.xls excel spreadsheet must be placed in the data directory, but can remain empty. For GPS data, the sheet must be entitled Mobile GPS Locations.

- Geographic coordinates for each bat file will be added to the latitude/longitude columns of the UserID output.

\section{Stationary Point Acoustic Monitoring}

- The latitude and longitude of each site must be entered

- Any higher data structure must also be entered

Table 5. Example stationary GPS Locations for running Bat2012 in the above data structure

\begin{tabular}{|c|c|c|c|}
\hline Grid Cell & Site & Latitude & Longitude \\
\hline Grid Cell1 & Site1 & 11.1111 & 22.11111 \\
\hline Grid Cell1 & Site2 & 11.2222 & 22.22222 \\
\hline
\end{tabular}

\section{Sunset/Sunrise Times}

- For sunset/sunrise the sheet must be named Sunset and Sunrise Times.

- For each Site/Date, enter the appropriate Sunset and Sunrise Times in 24-hr clock notation format

Table 6. Example sunset and sunrise times for running Bat2012 in the above data structure:

\begin{tabular}{|c|c|c|c|c|}
\hline Grid Cell & Site & Date & Sunset & Sunrise \\
\hline Grid Cell1 & Site1 & 20120601 & $20: 45$ & $05: 45$ \\
\hline Grid Cell1 & Site1 & 20120602 & $20: 46$ & $05: 43$ \\
\hline Grid Cell1 & Site2 & 20120701 & $20: 35$ & $06: 15$ \\
\hline
\end{tabular}




\section{Steps 2 and 3. Noise Scrubbing and Auto ID}

Analyzing data with EchoClass is straightforward as there are few options that can be selected by the user. This was done to improve accuracy, repeatability, and consistency in the results among datasets. When the EchoClass software is launched, the user interface in fig. 3 appears.

BAT_ID

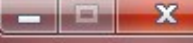

Select Data Directory

No directory selected

Browse

- Choose Species Set

(C) Set 1

Set 2

EPFU MYLE

LANO MYLU

LABO MYSE

LACI MYSO

PESU

LABO MYSE

LACI MYSO

MYAU NYHU

MYGR PESU

Set 3
EPFU MYAU
LANO NYHU
LABO
LACI

- Supplemental Data Options

GPS Information

Sunset/Sunrise Times

Process Data

Figure 3. Startup user interface for EchoClass software (from Britzke, 2012).

1. Select the Browse button and navigate to the appropriate directory (see examples above).

Chose the appropriate species set. If a species is not listed in a set it will not be an option for identification in this analysis. Thus, if that species is present it will be misclassified as a species that is listed. Because of this, the user should include all possible species which might be present in the area.

2. Click GPS information if the GPS data are provided in a specific format (table 5). This will result in location information being included in the output file.

3. Click Sunset/Sunrise Times if you want the activity metrics to be calculated. The user must provide the sunset and sunrise times for each night for the given location (table 6). This information will also be reported in the results output.

4. Click the Process Data button.

As the program runs a series of popups will appear and disappear. Once completed, a Microsoft Excel file titled "User ID Report" will be created in your selected working directory (the folder selected in with the browse button).

\section{Supplemental Data Options}

For the following options to function correctly, the GPS_and_Sunset_Master.xls Excel spreadsheet must be placed in the data directory selected to run the program on (that is, if Project1 is selected, the excel sheet must be located there as well).

\section{Step 5. Submit to NABat}

See the Introduction of this report for the steps for submitting data to NABat. To easily identify voucher files that should be prioritized for upload through your NABat Project page in EchoClass, sort, select, and save manually-vetted files. 


\title{
Chapter 5. Processing Zero-Cross Acoustic Data Using AnaLookW 4.2n (March 16, 2017)
}

\author{
By Cori Lausen²
}

${ }^{2}$ Wildlife Conservation Society Canada

The following chapter describes a recommended workflow using AnaLookW for processing acoustic data for the North American Bat Monitoring Program (NABat) where data were collected or converted to zero-cross files (.zc file format).

\section{Step 1. Attribute Files}

1. Ensure that minimum attribute data are included for each file. (See step 1 in the Introduction of this report for more detail on attribution data).If waypoints are not already included in your files, add GPS coordinates to stationary point acoustic data using Anahead or to transect datasets using GPS Integration Wizard prior to analysis.

- Using Anahead (Click on Tools -> Anahead); select your folder and highlight all the files; positions; enter manually; enter the latitude and longitude in World Geodetic System 1984 (WGS84) datum.

2. (OPTIONAL, but recommended) Sort your data into nightly subfolders. This is generally advantageous because the stationary point acoustic data for NABat are analyzed by species presence per detector per night. For example, one potential hierarchical folder organization scheme includes assigning a single folder per GRTS ID with subfolders for each detector and mobile transect. Within each detector subfolder there should be nightly subfolders.

- If you used a Titley Scientific detector, this is most easily achieved at the time of download (for example CFCRead for Anabat has a check box for "split nights").

- If you are using an auto ID software prior to using AnaLookW, many programs give you the option to sort your files by "bat-night."

- Any dataset can be split up by night using Tools => Folder $=>$ Split Folder in AnaLookW.

3. Add Location data to headers of files if this information is not already in the file metadata. It is highly recommended that you carry out this step even if this information is present in the filenames.

- In AnaLookW, perform the following for each folder: FILE $=>$ Mark All Files, EDIT =>Global Header Change. Add into the LOC field the Grid Cell GRTS ID and Site Name. For Transect folders, add Grid Cell GRTS ID Transect.

- If you know how to run a scan, you can send each detector's files (all nightly subfolders) through a single filter scan using a "blank" filter so that all files are listed in a list file (virtual subfolder, much like a playlist in iTunes). You can then open the resulting .anl file in AnaLookW and do a global header change on all files here, thus putting info into the header of all files in all nightly subfolders, instead of having to do each folder separately.

\section{Step 2. Noise Scrubbing}

Follow these next steps to remove non-bat files from your dataset.

\section{Option 1: Kaleidoscope or Kaleidoscape Pro}

- Run the files through Kaleidoscope or Kaleidoscope Pro (KSPro). You do not need to do any file conversion or auto ID, simply select to move noise files out of the dataset. Be sure to select the appropriate criteria for the noise scrubber (see KSPro workflow). 


\section{Option 2: AnaLookW}

- In AnaLookW, apply an "AllBats" or customized AntiNoise filter (see appendix B "Using Filters in AnaLookW"). Run a scan (Filter $=>$ Scan) using the selected filter across a copy of the dataset (folder tree), setting the output to generate a list file (only check the ANL list box). By opening the resulting .anl file in AnaLookW File Selector, you can mark all the files which passed the filter, reverse the marks and then delete (or move) all the files now marked (the files which didn't pass the filter).

\section{Step 3. Auto ID}

Use one of the following three options to obtain ZC files with species labels, using one or more of the following options:

\section{Option 1: Species Labels are Embedded in Files as MetaData During the Auto ID Process}

\section{SonoBat}

- SonoBat is used for files that start as full spectrum. SonoBat will place a species label in the file metadata that should appear in AnaLookW once the full spectrum file is converted to ZC format (see next step). The auto ID label will be in the Species field of the Header data; or if you have selected for SonoBat to change the filename, then the species label will appear at the end of the filename. In the latter case, it is desirable to leave the filenames with SonoBat Species Labels as this may be the only record you will have of what this auto ID program assigned to a file. Subsequent manual vetting in AnaLookW will place metadata into the species field that will override the auto ID in the final results file.

- After running the auto ID process in SonoBatch, you must convert full spectrum files to ZC files in order for them to be read in to AnaLookW. Do this in KSPro, BCID, or in newer versions of AnaLookW (TOOLS $=>$ CONVERTER $=>$ WAV TO ANABAT). Note: Appended species labels in filenames from SonoBat are scrubbed off the end of the filename in KSPro 4.3.2.

\section{Kaleidoscope Pro}

- Kaleidoscope Pro (KSPro) can be used for any recording file type. Before processing files with the auto ID option, check the appropriate file type checkboxes in the Batch tab. (for example, wav as input, ZC as output). The auto ID process will place a species label in the ZC file metadata which will be seen in the Species field of the Header data once in AnaLookW. There will also be information in parentheses about the auto ID (for example matching pulses; alternate IDsthis will depend on the version of KSPro that has been used).

\section{Bat Call Identification (BCID)}

- BCID can be used for any recording file type. If you use full spectrum files, you will need to first convert them to ZC within the program. The auto ID process of BCID will place a species label in the ZC file metadata (in the Species field of the Header data, appended with -bcid). (see chapter 3. Processing Acoustic Data Using Bat Call Identification (BCID ver.2.7d)).

\section{Option 2: Species Labels Are Embedded in Files as Metadata Following Application of Filters}

- Follow instructions in appendix B Additional "Guidance for AnaLookW." 


\section{Option 3: Species Labels Are Provided in an Excel Spreadsheet}

- The Excel spreadsheet provided as output from auto ID software (for example spreadsheet produced by EchoClass-see the EchoClass workflow in chapter 4) can be referred to during manual vetting of the files using AnaLookW as a viewer. Manual vetting will entail making changes in the Excel spreadsheet after viewing each file.

\section{Step 4. Manual Vetting}

To carry out manual vetting, follow the ruleset laid out in step 4 (after the Introduction) of this report. If you obtained files via Option 3 above, view each file in AnaLookW and adjust species labels as appropriate in the Excel spreadsheet. If you obtained files using Options 1 or 2, proceed with the following steps for manual vetting.

1. Ensure the Species List is visible. Check the option in View $=>$ Species List. This will allow you to add a species label to the metadata of each file by clicking a single button. Load or create a species list using one of the following options.

- Option 1: Load a Species List

- Click the LOAD button to the right of the species label buttons.

- Option 2: Create or edit a Species List

- Right-click each button and enter a 4-letter species code (table 3) or up to 8 letter frequency group or high/low class label (table 4).

- Select SAVE AS to save your species list.

- Tips for Species Lists

- Include group labels appropriate for your bat community (table 4).

- Prefix lowercased letters on species codes so it is possible to differentiate manually vetted labels, $m$, from auto ID labels (for example, "mLANO" for label assigned to a file identified as Lasionycteris noctivagans through manual vetting).

2. Load a file from your dataset. If your files are organized by detector and by nightly subfolders, start with night one of detector one.

3. FILE $=>$ DISPERSE.

1. Choose one of the following methods.

- Method 1: Select current folder. This will create a sorted .anl file just for the nightly folder you have selected. You will need to repeat this step for each night's folder for each detector.

- Method 2: Select a higher order folder. Include subfolders (check this box). For example, you might select to do a "disperse" of one detector. This means all nightly subfolders will be included in the "disperse" and you can view all recordings of this detector as though they were in one virtual folder together. This method involves fewer steps overall than Method 1, but you will have to pay attention to what nightly subfolder you are in during the vetting process.

2. Check the option for List File (.anl). Check to IGNORE PREFIXES. Then RUN. A new online software www.Anacraft.online will be available in 2018 which will facilitate multiple auto ID programs to be used on ZC recordings. This process will be different in this new program.

4. "Shift I" (or FILE => OPEN HERE) and click on Sorted.anl in whichever folder you directed the Disperse to start. (Sometimes you need to collapse directory and open it again to see this file-little bug! You close and reopen the folder in the left viewing pane using the \pm button by folder name.)

5. Using CHOOSE FIELD, review each of the species categories. The goal is to manually verify at least one file per night for each species. Recall that the label you place into the Header Species field when you manually vet should be prepended with a lower case letter " $m$ " (for manual review). Any file with an " $m$ " before the species will assume to have been verified manually. 


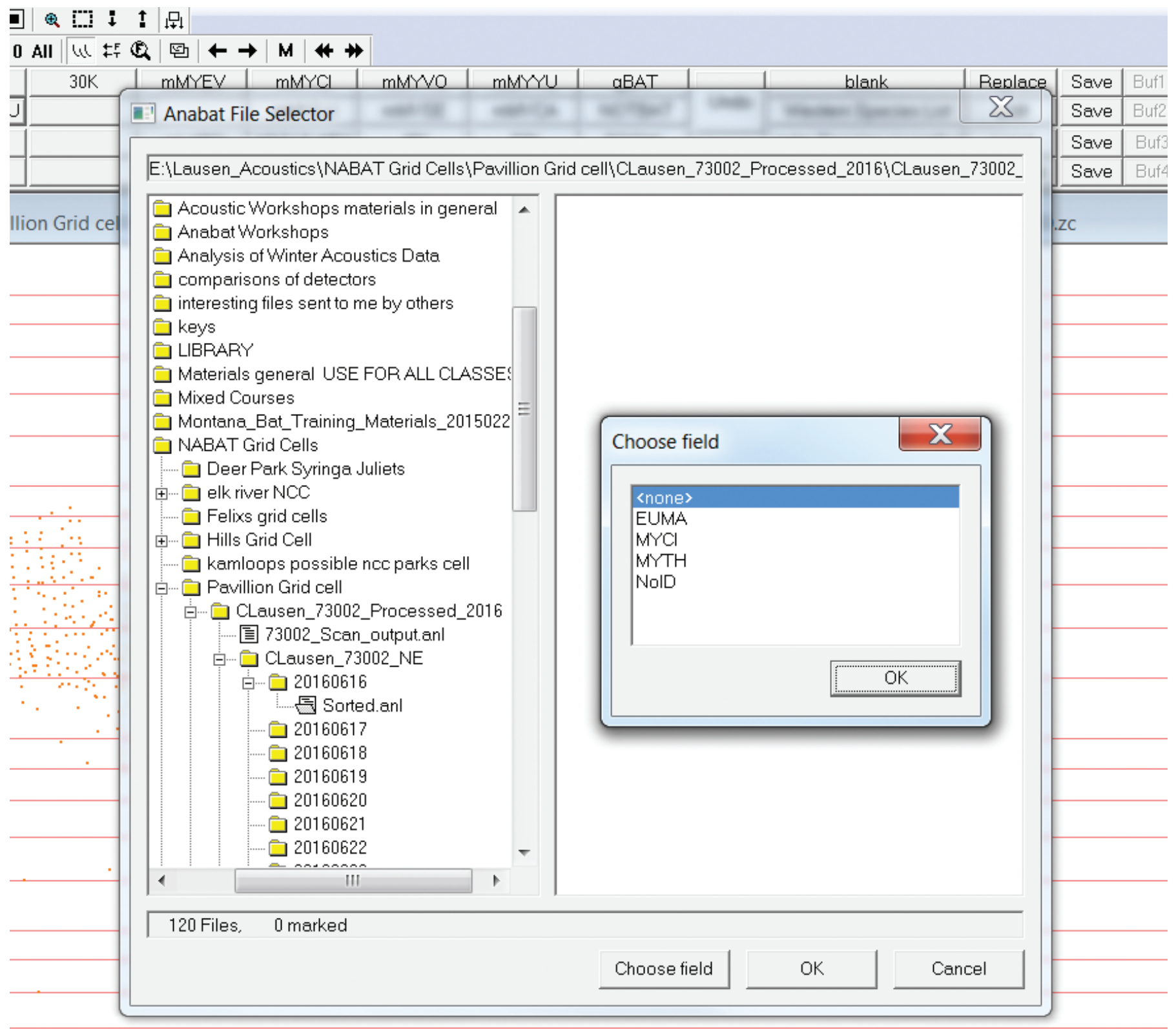

Figure 4. View of folder directory in AnaLookW.

- You can also use species couplet, group or class labels up to 8 letters, such as EPFULANO, LowF, and so forth. (see tables 3 and 4 for more labels). Since these types of labels are not used by the auto ID software, it will be clear that these labels were added during manual vetting.

- Recall that you can add multiple labels separated by a comma, with no spaces. This happens automatically when you press more than one species list button in the same file.

6. Repeat steps 3-5 until all nights of all detectors have been analyzed.

For stationary point acoustic data, you need only confirm one file of each species each night. For mobile transect acoustic data, all bat files should be reviewed.

- Required for Mobile Transect data and recommended for stationary point acoustic data: reviewing the "No_ID" field. These files did not get an auto ID and if you used filters, also did not get triggered by a filter.

- Why are some files No_ID? Files assigned the label "No_ID" are likely poor quality, high clutter recordings. However, with auto ID a file is occasionally labelled as "No_ID", even though good quality, identifiable pulses are present. This 
can occur when the recorded pulses have characteristics of more than one species. However, this can also occur if two, equally well-recorded species occur in the same file. Identifying high quality recordings of two species in the same file is especially important if there are species that you think are present in your area, but have not been detected in a certain night's dataset. In stationary point acoustic data, this may be the only reason to view "No_ID" bat passes.

\section{Mobile Transect Data Only}

The goal for collecting mobile transect acoustic data for NABat is to evaluate relative species abundance. Therefore, ensure that each bat file receives a label. If there are few "No_ID" files, then you may choose to go through all of them. If there are a lot of these files (for example, $>100$ ), you may instead opt to run a few filters to group the "No_ID" files into broader categories for easier vetting. For example, you could choose to run a general "40kMyotis" filter if you have one of these available. LOAD FILTER, then FILE $=>$ MARK IF PASS FILTER. Remember that once a filter has marked files, you can quickly jump from marked file to next marked file using $-/=$ keys and select on the species button at the top to add the label "40kMyo" (or use GLOBAL HEADER CHANGE and label "f40kMyo").

\section{Download Results into an Excel Spreadsheet Format.}

1. TOOLS $=>$ ANAHEAD

2. Select the folder in your dataset you want to summarize

3. $\quad$ FILE $=>$ DOWNLOAD.

4. Repeat 1 and 2 for all subfolders in the dataset.

5. In Windows Explorer, open up each Header.txt file that has been created in each folder/sub-folder, one at a time and copy/ paste the output into an Excel spreadsheet.

\section{Step 5. Submit to NABat}

See the Introduction of this report for the steps for submitting data to NABat. To easily identify voucher files that should be prioritized for upload through your NABat Project page using AnaLookW, use "Mark if Labelled" and select manually-vetted files.

\section{References}

Bat Call Identification, Inc., 2015, Bat Call Identification software (ver. 2.7d): Kansas City, Mo., accessed on 03 16, 2018, at http://www.batcallid.com/.

Britzke, E., 2012, Instructions for using the EchoClass acoustic program (ver. 3.0): U.S. Army Corps of Engineers, Research and Development Center, accessed on March 16, 2018, at https:/www.fws.gov/midwest/endangered/mammals/inba/surveys/ inbaAcousticSoftware.html.

Lausen, C.L., Burles, D., Blejwas, K., Nagorsen, D., Govindarajulu, P. and Friis, L., 2016, Long-eared bat taxonomy-Nuclear genetic evidence eliminates the species status of Keen's Myotis (Myotis keenii) [poster], Joint Meeting of Society for Northwest Vertebrate Biology and Washington and Idaho Chapters of The Wildlife Society, Coeur d'Alene, Idaho, Feb. 23-24, 2016. Bethesda, The Wildlife Society, accessed on March 16, 2018, at http://wildlife.org/.

Loeb, S.C., T.J. Rodhouse, L.E. Ellison, C.L. Lausen, J.D. Reichard, K.M. Irvine, T.E. Ingersoll, J.T.H. Coleman, W.E. Thogmartin, J.R. Sauer, C.M. Francis, M.L. Bayless, T.R. Stanley, and D.H. Johnson. 2015. A plan for the North American Bat Monitoring Program (NABat) (p. 112). United States Department of Agriculture, Forest Service, Research \& Development, Southern Research Station.

NABat Acoustic Data Analysis Workflow Workshop, 2017, U.S. Geological Survey, Fort Collins Science Center, Fort Collins, Colo., September 6-8, 2017.

Szewczak, J., and Szewczak, N., 2017, SonoBat ver. 4.2.1: Arcata, Ca. Sonobat, accessed March 21, 2018, at https://sonobat.com/.

Titley Scientific, 2016 AnalookW software ver. 4.2n (16 March 2017): Columbia, Mo.,Titley Scientific, accessed on March 21, 2018, at https://www.titley-scientific.com/us/. 


\title{
Appendix A. Case Study-Using Rule Sets to Process Acoustic Data for USDA Forest Service Lands in Northern California
}

\author{
By Ted Weller ${ }^{3}$
}

${ }^{3}$ USDA Forest Service

The standard for which files should be considered for manual vetting will vary by location according to how common a species is expected to be and how likely it is to be confused with other species at the site. Location-based rulesets can be established to direct manual vetting towards files that require the most attention (that is, unusual species, files where SonoBat has lower levels of certainty in its auto ID). Rulesets can be codified to automatically assign species to files for which SonoBat has high levels of confidence, and assign files to frequency groups (for example HiF, Q40k) where SonoBat has low confidence or gleaned little information from the file. When establishing rulesets, take into consideration the expected probability of a species' detection in deciding how to direct manual vetting efforts. For example, at some sites, a single species can comprise greater than or equal to $(\geq) 50$ percent of the total files recorded. In this case, confirmation of this species can be based on lower levels of certainty by SonoBat (for example, TABR 2 of 2) than we would normally accept. Rulesets are plastic and evolve over time to improve accuracy of species identification and, importantly, improve efficiency with which assignments to a species or species group are made. Rulesets begin as regional tools and evolve into sub-regional tools as we learn more about the relative probability of species occurring in the parts of the region studied. When appropriate, rulesets can be established for individual sites. In northwest California for example, two sites approximately 100 kilometers $(\mathrm{km})$ apart have different rulesets. At one site, TABR is exceedingly common and LANO is less common, while at the other site, TABR is very uncommon and LANO is common.

Hence we review few of the potential TABR files and more LANO files at the first site and all TABR files and very few LANO files at the second site. This saves time at both sites.

In the following, we describe a process for using established rulesets to determine activity levels by species and species groups by night in mountainous areas on USDA Forest Service land in northern California.

1. Vetting table is exported as.txt file, selecting output null as "“".

2. Exported .csv file is opened in EXCEL and a Pivot Table is inserted to summarize the data. 


\begin{tabular}{|c|c|c|c|c|c|c|c|c|c|c|c|c|}
\hline$\Delta$ & A & B & $\mathrm{C}$ & D & $\mathrm{E}$ & $\mathrm{F}$ & G & $\mathrm{H}$ & I & J & K & L \\
\hline 1 & Site/Date & EPFU & LACI & LANO & MYCA & MYEV & MYLU & MYTH & MYvo & MYYU & TABR & Number of Species \\
\hline 2 & SE Lyon's Creek & & & & & & & & & & & 5 \\
\hline 3 & $6 / 12 / 2017$ & & & & & & & & & & & 0 \\
\hline 4 & $6 / 13 / 2017$ & & 1 & 1 & & & & & & & & 2 \\
\hline 5 & $6 / 14 / 2017$ & & 1 & & 1 & 1 & & & 1 & & & 4 \\
\hline 6 & $6 / 15 / 2017$ & & & 1 & 1 & & & & & & & 2 \\
\hline 7 & SW Granite Sprir & & & & & & & & & & & 7 \\
\hline 8 & $6 / 12 / 2017$ & & & & & & & & & & & 0 \\
\hline 9 & $6 / 13 / 2017$ & & & & 2 & & & 1 & & & 1 & 3 \\
\hline 10 & $6 / 14 / 2017$ & & & 1 & 5 & & 1 & 1 & & & & 4 \\
\hline 11 & $6 / 15 / 2017$ & 1 & & & 5 & 1 & & & & & & 3 \\
\hline 12 & NW Ice House & & & & & & & & & & & 7 \\
\hline 13 & $6 / 12 / 2017$ & & & & & & & & & & & 0 \\
\hline 14 & $6 / 13 / 2017$ & & 1 & 5 & 5 & 1 & 2 & & & 1 & & 6 \\
\hline 15 & $6 / 14 / 2017$ & & & 5 & 5 & & 5 & & & 2 & & 4 \\
\hline 16 & $6 / 15 / 2017$ & & 2 & 5 & 5 & & 5 & & 2 & 3 & & 6 \\
\hline 17 & NE Pond & & & & & & & & & & & 6 \\
\hline 18 & $6 / 12 / 2017$ & & & & & & & & & & & 0 \\
\hline 19 & $6 / 13 / 2017$ & & & 2 & 5 & 1 & & & & & & 3 \\
\hline 20 & $6 / 14 / 2017$ & & 1 & 3 & 5 & 1 & 2 & & & & & 5 \\
\hline 21 & $6 / 15 / 2017$ & 1 & & 5 & 5 & & 2 & & & & & 4 \\
\hline
\end{tabular}

Figure 5. A standard output from SonoBat summarized in Excel (Szewczak and Szewczak, 2017). 
The output table includes the number of files confirmed per night populating the species-by-night matrix. Values $\geq 1$ will ultimately be reduced to presence/not-detected values for analysis. Here they represent a crude, proposed method of assigning confidence to species ID per night.

The following rulesets were translated into a PYTHON script (https://github.com/brendan-ward/echoclean) and pointed at text output from a SONOBATCH run.

- For EPFU, LANO, MYCA, MYCI, MYLU, MYVO, MYYU, and PAHE, we will generally only review files if \#Maj=\#Accp $\geq 3$, files with \#Maj=4 and \#Accp=5, and \#Maj=5 and \#Accp $<=7$, and \#Maj=6 and \#Accp $<=8$ should also be reviewed.

- When ANPA, COTO, EUMA, EUPE, LABL, LACI, MYEV, MYTH, NYSP, or TABR are listed in the "1 ${ }^{\text {st" }}$ column, review all files (up to 10 files per night) until at least five files per species per night can be confirmed.

The PYTHON script creates a "Species" column and an "Inspect (Y/N)" column. The species column is populated with a label: species, couplet (for example EPFULANO), frequency group (for example, 50k), or high/low frequency class (for example, HighF) according to SONOBATCH output parameters. Files without adequate information from SONOBATCH are identified simply as "Bat". In some cases these have been found not to be bats, despite the file having been scrubbed in earlier steps. The relative proportion of files that are not bats appears to depend on site and season (for example, bird songs and insects can sometimes trick the scrubbers). If it is important to quantify the total number of files produced by bat echolocation ('mobile transect acoustic monitoring' for NABat, Loeb and others [2015]), low-quality and non-bat files are included in manual vetting efforts.

3. Files highlighted by ruleset (via the Python script) are prioritized for manual vetting.

- Output is sorted as follows:

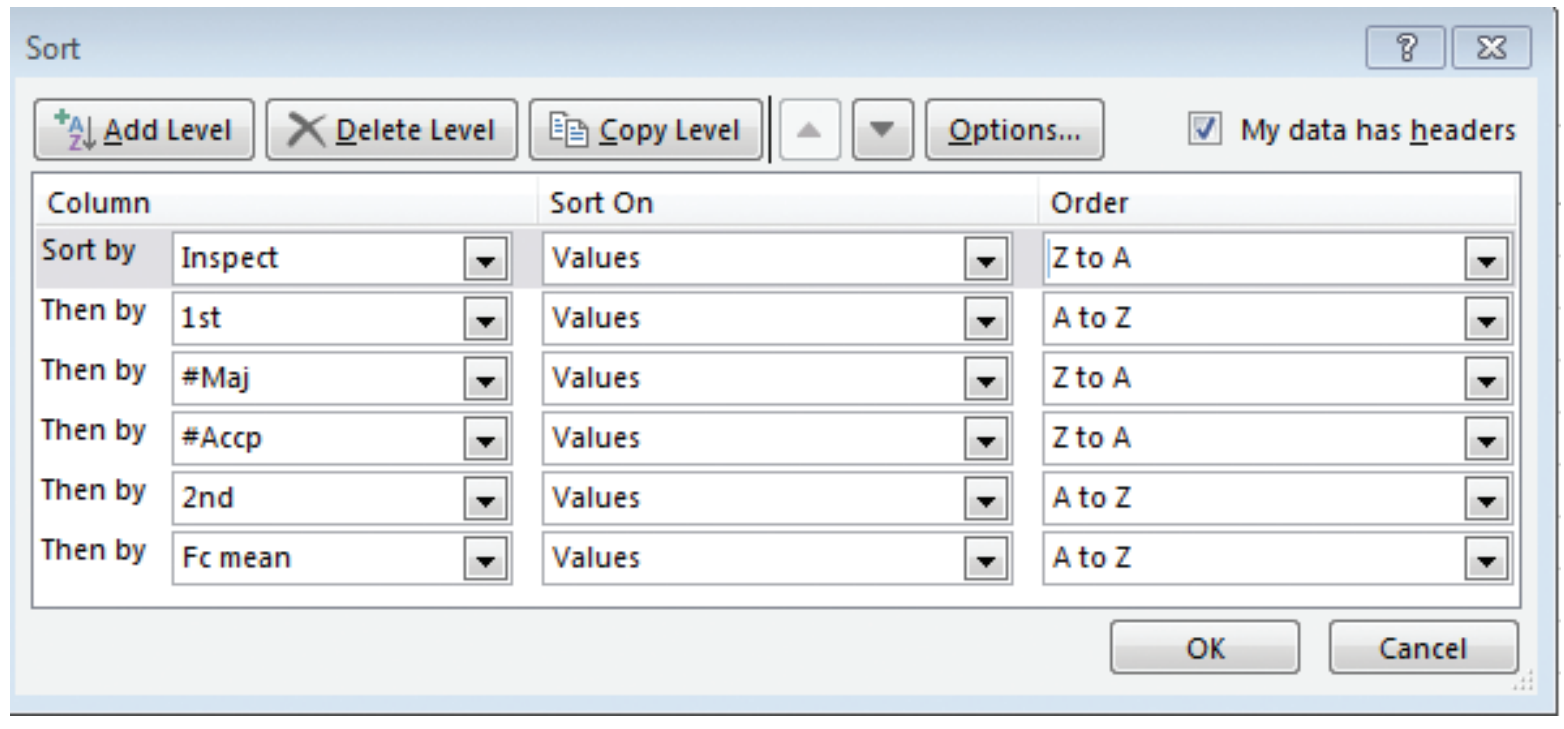

Figure 6. "Sort" criteria used to arrange summary output in Excel.

- Sorting orders the files to be manually vetted by potential species followed by probability that they will ultimately be assigned to that species and groups them according to other potential species in the file and frequency range.

- Each file with "Inspect=Y" is manually vetted and assigned to species or group. Manual vetting is based on comparison to reference files, regional guidance (with images) written by Ted Weller, and experience of the observer.

4. Following manual vetting, files are re-sorted excluding the "Inspect" column such that potential species ID's are listed in order of SonoBat's guidance.

- Patterns in eventual ID's compared to SonoBat ID's are studied to refine the ruleset for each of the species.

5. Night of recording is extracted from the filename and used in a pivot table to create a matrix of number of files assigned to each species or group for each night of recording. 


\title{
Appendix B. Additional Guidance for AnaLookW
}

\author{
By Cori Lausen² \\ ${ }^{2}$ Wildlife Conservation Society Canada
}

\section{Tips for using AnaLookW}

- TOOLS $=>$ OPTIONS $=>$ DISPLAY will allow you to change colors if needed.

- TOOLS $=>$ OPTIONS $=>$ change DELAY to $300 \mathrm{~ms}$ (so the user can scroll through files at reasonable speed by holding the closed square bracket or W keys) - recommended.

- Always save files to C drive (not to desktop or My Documents), as AnaLookW does not like files that become too buried in a directory and the desktop is actually an embedded location in your hard drive!

- Mark all files (FILE $=>$ MARKING $=>$ MARK ALL). You then can use the button REPLACE in the top of the AnaLookW screen to replace a field for all marked files (for example Highfreq instead of HighF). Recall that there is no "undo" if you make a mistake.

- To move from file to file that has a label in species field of header (for example skipping blank species), hold "Shift" and press the square bracket keys "[" or "]".

- To skip between marked files, use the "-“" and "=” keys

- Reverse marks as needed, in FILE => MARKING or "right-click" on file names in Anabat File Selector Window (Shift 1).

- FILE $=>$ MARK IF LABELLED (only mark those files with a species label).

- $\mathrm{EDIT}=>$ DELETE IF MARKED. (For example, you could "Reverse all marks" after marking bat files (so marked files are the noise files), then it is possible to move or delete files if you are confident they are all "noise" (non-bat recordings)

- You can use the "m" key on your keyboard to pull up a measuring screen. (or VIEW $=>$ MEASURES). If you use numeric parameters to help manually vet, you can view measurements of single pulses or averages of pulses on the screen using this " $m$ " key.

- You do not need to use the "Measures" screen to evaluate "Fmin" if there are many pulses, as this could be misleading. Instead, hold your curser at the bottom of the call of interest and look at the number on bottom right of the screen.

- Under VIEW $=>$ OFFDOTS you can choose to visualize your "offdots". Filters turn dots "off." By choosing to visualize "offdots" you can see which dots are not passing the filter criteria.

- You can view your file in a screen next to another screen with a reference file by selecting VIEW $=>$ TILE HORIZONTAL (or TILE VERTICAL). You can then link both screens so they maintain the same settings by selecting the link screen button that looks like two overlapping squares. The screen you are in at the time of clicking the link screen button becomes the "Master" screen while the other screen becomes its "Slave." Alternatively, you can link them both and have both as "Masters."

- If at any point the species field is too full (for example, when Kaleidoscope Pro fills in a lot of information in parentheses after a space following the species ID label), you can move this information to the "Notes" field using COPY $=>$ PASTE.

- **IMPORTANT** It is critical that the auto ID labels be retained and remain in the "Species Field" so that these data are retained through to the final spreadsheet. 
- "Buffer" is located to the far right-hand side of the species list. Use this if you want to have a "push button" way of adding a label to the "Species Field" that is, more than eight characters long. You can only have two buffer labels (top two) that will add to a "Species Field" when clicked. Enter a label into the "Buffer" by first typing into the "Species Field" exactly what you want to save into the "Buffer". Then click the "Save" button next to the "Buf1+" or "Buf2+" buttons. The "Buf" button is now lit and ready to use as another "Species Label".

\section{Using Filters in AnaLookW}

1. Obtain AnaLookW filters. It is recommended that only Strict or "Diagnostic" type species filters be used. (If you are simply scrubbing out noise files from bat files, then an "AllBats" filter can be used.)

- Filter files (.abf files) can be obtained from trusted sources, or you can create a filter by filling in appropriate parameters.

- If you are not an advanced user, then these parameters should be obtained from a trusted source. Parameters can be inserted into the filters using FILTER $=>$ NEW FILTER $=>$ SAVE FILTER.

2. Apply filter(s).

Choose one of the following options.

Option 1: You can apply a filter to any given folder of bat files (FILTER $=>$ LOAD FILTER, then FILE $=>$ MARKING $=>$ MARK IF PASS FILTER, then view marked files and (or) perform EDIT $=>$ GLOBAL HEADER CHANGE).

Option 2: Use a Scan to apply one or more filters at one time. Be sure to only select the output option "ANL". This produces a "scan file" (.anl file format). Recall that using a "Scan" instead of applying a filter on a per folder basis has the advantage of seeing all files of a dataset from all subfolders at once in one "virtual folder" (.anl file). This allows actions to be completed on the dataset all at once, despite the fact they are in separate subfolders (for example, nightly folders). "Scan" files (.abs) are required if you are going to scan with more than one filter at a time.

3. Once a filter/scan has been applied, you should put a species label into the Species field of the header so that you know this file has passed a species-level filter. You will use species labels such as fLABO. This means you used a filter to identify a file that is likely a LABO bat pass. The use of the lower case "f" is a good idea, as AnaLookW has the ability to ignore lower case initial letters in downstream analyses. The use of a prefix such as "f" or " $\mathrm{m}$ " is critical for NABat downstream analyses as it conveys how the species label was determined ( $\mathrm{f}=$ by filter; $\mathrm{m}=$ by manual vetting).

- If you applied a single filter to a folder (MARK IF PASS FILTER) then simply use GLOBAL HEADER CHANGE to apply a label (for example, fLABO for files that passed the LABO filter).

- If you applied a scan of a single or multiple filters, use the .anl file to view each field (generally each field is the name of the filter). For each field, MARK ALL, then GLOBAL HEADER CHANGE to insert a species label into the Header (for example, fLABO). Do for each field.

Conclusion: All files that passed a strict or diagnostic filter have now had a species label inserted into its metadata. You are ready to manually vet, see chapter 5, Step 4 . Manual Vetting. 


\section{Appendix C. Bat Auto ID Species Confusion Matrix}

This appendix provides a "Bat Auto ID Species Confusion Matrix" as a quick look-up guide indicating which species are commonly confused by auto ID software. In the matrix, red cells indicate species not currently included in classifiers used for automated species identification. Grey cells (along diagonal) are not applicable to the matrix.

Click on the link to open the Excel file: ofr2018-1068_appendixC_BatAutoID_SpeciesConfusionMatrix.xlsx 


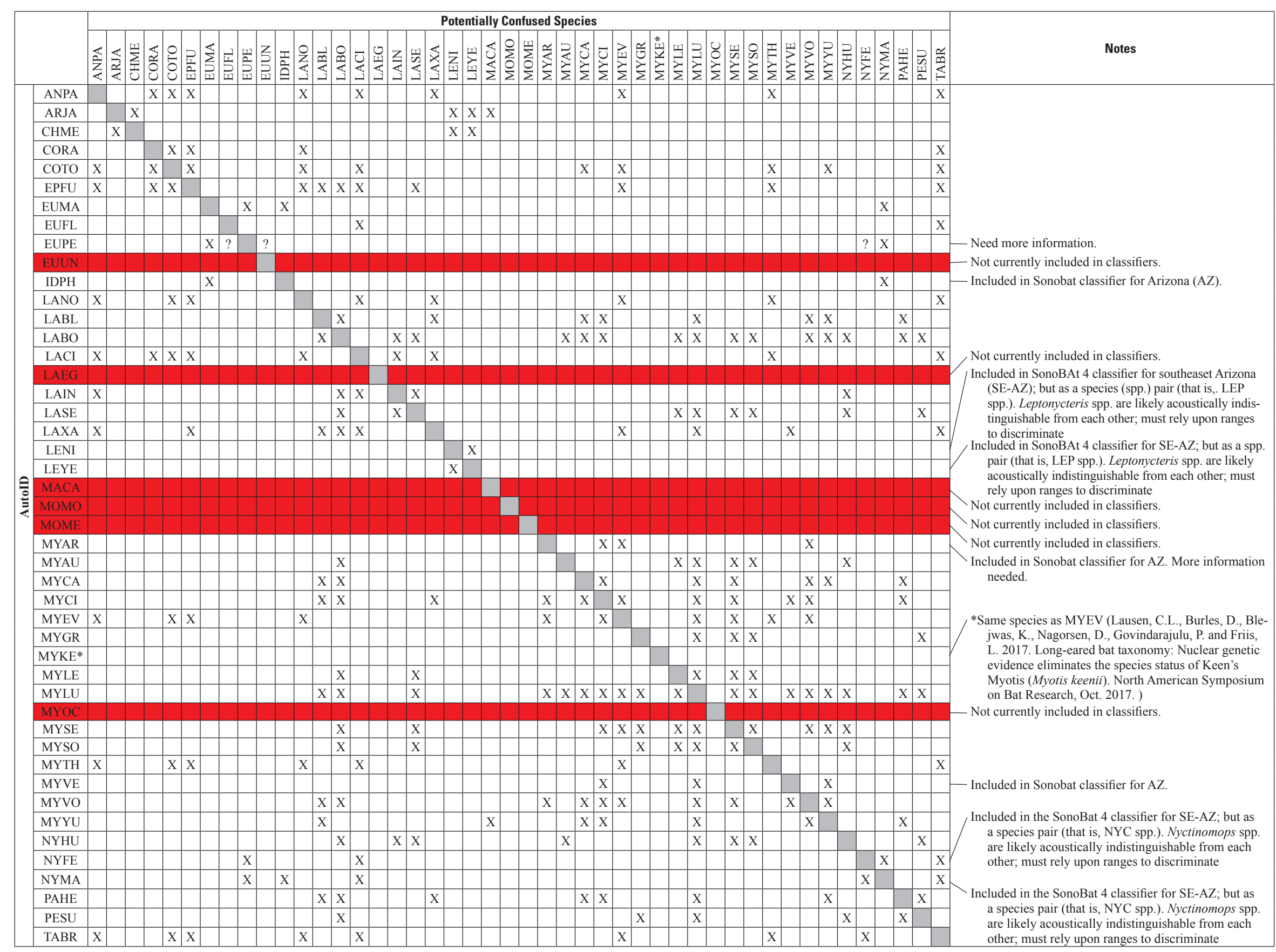


For more information concerning the research in this report, contact the Center Director, USGS Fort Collins Science Center

2150 Centre Ave., Bldg. C

Fort Collins, CO 80526-8118

(970) 226-9398

Or visit the Fort Collins Science Center website at https://www.fort.usgs.gov/ 
